\title{
MAGNETIC FIELD STRENGTHS IN THE HOT SPOTS AND LOBES OF THREE POWERFUL FANAROFF-RILEY TYPE II RADIO SOURCES
}

\author{
M. J. HardCastle AND M. Birkinshaw \\ Department of Physics, University of Bristol, Tyndall Avenue, Bristol BS8 1TL, UK \\ R. A. CAMERon AND D. E. Harris \\ Harvard-Smithsonian Center for Astrophysics, 60 Garden Street, Cambridge, MA 02138 \\ L. W. LOONEY \\ Max-Planck-Institut für extraterrestrische Physik (MPE), Garching, Germany \\ AND \\ D. M. WORRALL \\ Department of Physics, University of Bristol, Tyndall Avenue, Bristol BS8 1TL, UK \\ Received 2002 June 6; accepted 2002 August 29
}

\begin{abstract}
We have made deep Chandra observations of three powerful Fanaroff-Riley type II (FR II) radio sources: two quasars (3C 263 and 3C 351) and one radio galaxy (3C 330). X-ray emission from hot spots and lobes, as well as from the active nucleus, is detected in each source. We model the hot spots' synchrotron spectra using VLA, BIMA, and Hubble Space Telescope data. In 3C 263 and 3C 330, the hot spots' X-ray emission is at a level consistent with being synchrotron self-Compton (SSC) emission, with a hot spot magnetic field close to the equipartition value. In the two hot spots of 3C 351, however, an SSC origin for the X-rays would require the magnetic field strength to be an order of magnitude below the equipartition value in our models; in addition, there are offsets between the radio, optical, and X-ray emission from the secondary hot spot that are hard to explain in a simple SSC model. We discuss the emission mechanisms that may be responsible for these observations. On our preferred model, the X-ray emission from the radio lobes of the three sources is due to inverse Compton scattering of the microwave background radiation. If this is the case, the magnetic field strengths in the lobes are typically about a factor of 2 below the equipartition values, assuming uniform lobe electron and magnetic field distributions. We detect extended X-ray emission, which we attribute to a cluster/group environment, around 3C 263 and 3C 330. This detection allows us to show that the lobes are close to pressure balance with their surroundings, as long as no nonradiating particles contribute to the internal pressure of the lobes.
\end{abstract}

Subject headings: galaxies: active — galaxies: individual (3C 263, 3C 351, 3C 330) — quasars: general — radiation mechanisms: nonthermal $-\mathrm{X}$-rays: galaxies

\section{INTRODUCTION}

Chandra has now detected a large number of X-ray features related to the jets and hot spots of extragalactic radio sources (see Harris \& Krawczynski 2002 for a recent review). The jet detections have attracted a great deal of interest, but much of the fundamental physics behind them remains unclear. The X-ray jets commonly seen in FanaroffRiley type I (FR I) sources (Worrall, Birkinshaw, \& Hardcastle 2001a) probe the high-energy particle acceleration in these objects (Hardcastle, Birkinshaw, \& Worrall 2001b), while the few Fanaroff-Riley type II (FR II) jets that have been detected (e.g., Schwartz et al. 2000) may be evidence for extremely high bulk speeds on kiloparsec scales (e.g., Tavecchio et al. 2000). But the details of the processes responsible for producing the X-rays in both classes of source are still debatable, and the issue is confused by uncertainties about the Doppler boosting factors of the X-rayand radio-emitting material.

In hot spots the situation is more clear-cut. These structures, which are believed to trace a strong terminal shock or shocks at the end of the jet in powerful FR II sources, are generally thought to be unlikely to be moving with respect to the observer at highly relativistic speeds (Best et al. 1995;
Scheuer 1995; Arshakian \& Longair 2000), although moderately relativistic motions in and beyond the hot spot (with $v \sim 0.3 c$ ) may be required to explain some observations (Dennett-Thorpe et al. 1997). Their radio-to-optical spectra are often well constrained, giving limits on the amount of synchrotron emission expected in the X-ray, and their sizes and structures are often well known. This means that it is possible to make a simple prediction of the X-ray flux densities expected from inverse Compton emission. The dominant emission process for bright, compact hot spots that show strong spectral steepening or a synchrotron cutoff at high radio/optical frequencies is likely to be synchrotron self-Compton emission (SSC), in which the radio photons from the synchrotron process are inverse Compton scattered into the X-ray band by the synchrotron-emitting electrons. A prediction of the SSC flux density can be made if the magnetic field strength in the hot spot, and therefore the electron number density, can be estimated. Conversely, a measured X-ray flux density that is inferred to be SSC can be used to estimate the magnetic field strength in the hot spot.

ROSAT observations of the archetypal FR II object Cygnus A (Harris, Carilli, \& Perley 1994) showed that the $\mathrm{X}$-ray emission from its hot spots was consistent with the 
TABLE 1

SSC and Unknown High-Energy FR II Hot Spot Detections

\begin{tabular}{ccccc}
\hline \hline Mechanism & Source & Source Type & Instrument & Reference \\
\hline SSC near equipartition.............. & Cygnus A & NLRG & ROSAT & 1 \\
& & & Chandra & 2 \\
& 3C 295 & NLRG & Chandra & 3 \\
& 3C 123 & NLRG & Chandra & 4 \\
& 3C $196^{\mathrm{a}}$ & $\mathrm{Q}$ & HST & 5 \\
& 3C 207 & $\mathrm{Q}$ & Chandra & 6 \\
& 3C 263 & $\mathrm{Q}$ & Chandra & 7 \\
& 3C 330 & NLRG & Chandra & 7 \\
Unclear & 3C 351 & Q & Chandra & 7,8 \\
Non-SSCE................................................... & Pictor A & BLRG & Einstein & 9 \\
& & & Chandra & 10 \\
& 3C 390.3 & BLRG & ROSAT & 11,12 \\
& 3C 303 & Q & ROSAT & 13 \\
\hline
\end{tabular}

Note.-Source type abbreviations are as follows: NLRG = narrow-line radio galaxy; $\mathrm{BLRG}=$ broad-line radio galaxy; $\mathrm{Q}=$ quasar

a Claimed optical SSC detection.

b This source will be discussed in more detail below.

${ }^{c} \mathrm{X}$-ray source may be a background quasar.

REFERENCES.- (1) Harris et al. 1994; (2) Wilson et al. 2000; (3) Harris et al. 2000; (4) Hardcastle et al. 2001a; (5) Hardcastle 2001; (6) Brunetti et al. 2002a; (7) this paper; (8) Brunetti et al. 2001; (9) Röser \& Meisenheimer 1987; (10) Wilson et al. 2001a; (11) Prieto 1997; (12) Harris et al. 1998; (13) Hardcastle \& Worrall 1999.

SSC process if the magnetic field strength was close to the equipartition or minimum-energy values (Burbidge 1956). This result is often taken as evidence for equipartition between magnetic fields and synchrotron-emitting electrons in radio sources in general. Other ROSAT observations, however (e.g., Harris, Leighly, \& Leahy 1998), showed that some hot spots had X-ray emission too bright to be produced by the SSC mechanism with equipartition field strengths, suggesting that a different emission process was responsible. At the time of writing, after a number of new Chandra hot spot detections, this dichotomy remains, as shown in Table 1. Three interesting facts about this division are immediately apparent. First, the number of objects whose emission processes are not from SSC near equipartition (hereafter "non-SSCE" objects) is still a significant fraction of the total. Second, the non-SSCE objects all display broad emission lines, which may, after all, indicate some role for beaming in the hot spots. And, third, the nonSSCE objects all have optical synchrotron hot spots.

The major remaining questions in this area are therefore the following:

1. Are magnetic field strengths close to equipartition typical in hot spots?

2. What emission process is responsible for non-SSC sources, and is it related to their other common properties, or to relativistic beaming?

Question 1 can be addressed only by new observations of hot spots, and in the first part of this paper we report on our deep Chandra observations of three sources selected on the basis of bright compact radio hot spot emission, describing the emission from their hot spots, lobes, nuclei, and cluster environments. In $\S 7$ we will return to the second question.

The remainder of the paper is organized as follows. In $\S 2$ we discuss the selection of the sample and the observations we have used. In $\S 3$ we discuss the general methods of analysis we have applied. In $\S \S 4,5$, and 6 we discuss the results of applying these methods to our three target sources.
Finally, in $\S \S 7$ and 8, we explore the general conclusions that can be drawn from our results.

Throughout the paper we use a cosmology with $H_{0}=65$ $\mathrm{km} \mathrm{s}^{-1} \mathrm{Mpc}^{-1}, \Omega_{m}=0.3$, and $\Omega_{\Lambda}=0.7$. Spectral indices $\alpha$ are the energy indices and are defined throughout in the sense $S \propto \nu^{-\alpha}$. J2000.0 coordinates are used throughout.

\section{SAMPLE, OBSERVATIONS, AND DATA REDUCTION}

\subsection{Sample Selection}

We selected our target objects from the 3CRR sample (Laing, Riley, \& Longair 1983), not because the selection properties of that sample were particularly important to our scientific goals, but because the sample members at $z \leq 1$ have almost all been imaged in the radio at high resolution and with good sensitivity. As a consequence, the properties of the hot spots in this sample are well known. We selected three objects with bright, compact hot spots: 3C 263, 3C 351, and 3C 330. To predict an approximate Chandra hot spot count rate, assuming equipartition, we used a simple SSC model for each source and then proposed observations that would allow a high-significance detection of the hot spots unless the magnetic field was much greater than the equipartition value. The general properties of our target sources are listed in Table 2.

\subsection{X-Ray Observations}

The three targets were observed with Chandra, as shown in Table 3. The standard ACIS-S observing configuration was used for 3C 263 and 3C 330. For 3C 351 we used a standard half-chip subarray and read out only four chips, reducing the frame time to $1.74 \mathrm{~s}$, in order to reduce pileup in the bright X-ray nucleus. Subsequent processing was carried out using CIAO 2.2 and CALDB 2.9. All three observations were processed identically. The initial processing steps consisted of removing high-background intervals (significant 
TABLE 2

Properties of the OBSERVEd SAMPLe

\begin{tabular}{|c|c|c|c|c|c|c|}
\hline Source & $z$ & $\begin{array}{c}\text { Scale } \\
\left(\mathrm{kpc} \operatorname{arcsec}^{-1}\right)\end{array}$ & $\begin{array}{c}S_{178 \mathrm{MHz}} \\
\text { (Jy) }\end{array}$ & $\begin{array}{c}\text { Largest Angular } \\
\text { Size } \\
(\operatorname{arcsec})\end{array}$ & $\begin{array}{l}\text { Linear Size } \\
\quad(\mathrm{kpc})\end{array}$ & $\begin{array}{c}\text { Galactic } N_{\mathrm{H}} \\
\left(\times 10^{20} \mathrm{~cm}^{-2}\right)\end{array}$ \\
\hline 3C $263 \ldots \ldots \ldots \ldots$ & 0.6563 & 7.5 & 16.6 & 51 & 380 & 0.91 \\
\hline 3C $330 .$. & 0.5490 & 6.9 & 30.3 & 60 & 410 & 2.94 \\
\hline 3C $351 \ldots \ldots \ldots \ldots . . . . .$. & 0.371 & 5.5 & 14.9 & 74 & 410 & 2.03 \\
\hline
\end{tabular}

Note.-Hydrogen column densities for 3C 263 and 3C 351 are taken from Lockman \& Savage 1995; for 3C 330 the quoted value is interpolated from the database of Stark et al. 1992.

for 3C 263 and 3C 351) and generating a new level 2 events file with the latest calibration applied and with the 0.5 pixel randomization removed. After aligning the $\mathrm{X}$-ray and radio cores to correct for small offsets between the radio and $\mathrm{X}$-ray images, which we attribute to uncertainties in the Chandra aspect determination (discussed in more detail below), we used smoothed images made in the $0.4-7.0 \mathrm{keV}$ bandpass to define extraction regions for the various detected X-ray components.

\subsection{Radio Data}

Radio observations are required in order to model the expected SSC emission from the hot spot. When it became apparent that the small-scale structure of 3C 263's hot spot was not well enough constrained for our purposes, the Very Large Array (VLA) schedulers kindly allowed us to make a short observation of the source at $15 \mathrm{GHz}$ in A configuration. In addition to this new data set, we obtained $u v$ data sets for all sources from the archives with the permission of the original observers, while some other observers supplied us with maps or $u v$ data directly. A complete list of the VLA observations we used is given in Table 4. In all cases where we reduced the data ourselves, standard procedures were followed within AIPS, using several iterations of phase selfcalibration sometimes followed by one amplitude selfcalibration step. Errors in the fluxes derived from the VLA data are dominated in all cases by the uncertainties in primary flux calibration, nominally around $2 \%$.

\subsection{Millimeter-Wave Observations}

Observations in the millimeter band are also useful to this work, as electrons with energies around the low-energy cutoff inferred in some hot spots $(\gamma \sim 1000)$ scatter photons at millimeter wavelengths into the X-ray. We therefore observed all three of our targets with the Berkeley-IllinoisMaryland Association (BIMA) array (Welch et al. 1996).
The observations were made in the $\mathrm{B}$ configuration of BIMA, using two $800 \mathrm{MHz}$ channels centered on 83.2 and $86.6 \mathrm{GHz}$, which were combined to give an effective observing frequency of $84.9 \mathrm{GHz}$. Observing times and dates are given in Table 5. The data were reduced in MIRIAD, and final imaging was carried out within AIPS. In all cases the target hot spots were detected; $85 \mathrm{GHz}$ radio cores were also detected in 3C 263 and 3C 351. The resolution of BIMA in this configuration and frequency is $\sim 3^{\prime \prime}$, so the observations allow us to distinguish between hot spot and lobe emission and to separate double hot spots but not to comment on structure in individual hot spots. The BIMA images will be presented elsewhere.

\subsection{Optical Observations}

For optical information, which tells us about the highenergy synchrotron spectrum, the archival Hubble Space Telescope (HST) WFPC2 data sets listed in Table 6 were used. Cosmic-ray rejection was performed (where necessary) within IRAF, and flux densities or upper limits were measured using standard small-aperture photometry techniques in AIPS. As with the X-ray data, the optical data were aligned with the radio data by shifting the optical coordinates to align the radio nucleus with the optical peak. We discuss residual astrometric uncertainties in more detail below.

\section{ANALYSIS METHODS}

\subsection{X-Ray Spectral Extraction}

We detected $X$-ray emission from the nucleus, from one or more hot spots, and from the radio lobes of each source, as shown in Figure 1 and discussed in the following sections. For each of these components, spectra were extracted (from the regions listed in Table 7 and illustrated in Fig. 2) using

TABLE 3

Chandra OBSERVATIONS

\begin{tabular}{|c|c|c|c|c|}
\hline Source & Date & $\begin{array}{l}\text { Live Time } \\
\text { (s) }\end{array}$ & $\begin{array}{l}\text { Effective Time } \\
\text { (s) }\end{array}$ & $\begin{array}{l}\text { Observatory } \\
\text { Roll Angle } \\
\text { (deg) }\end{array}$ \\
\hline 3C $263 \ldots \ldots \ldots \ldots$ & 2000 Oct 28 & 49,190 & 44,148 & 37 \\
\hline 3C $351 \ldots \ldots \ldots \ldots \ldots$ & 2001 Aug 24 & 50,920 & 45,701 & 254 \\
\hline 3С $330 \ldots \ldots \ldots \ldots \ldots$ & 2001 Oct 16 & 44,083 & 44,083 & 319 \\
\hline
\end{tabular}

NoTE.-The effective time quoted is the livetime after high-background filtering. The satellite roll angle $\theta_{\text {roll }}$ determines the position angle on the sky of any readout streak; the position angles (defined conventionally as north through east) are $360^{\circ}-\theta_{\text {roll }}, 180^{\circ}-\theta_{\text {roll }}$. 
TABLE 4

VLA ObServations Used in the Analysis

\begin{tabular}{|c|c|c|c|c|c|c|c|}
\hline Source & Program ID & Date & $\begin{array}{l}\text { Frequency } \\
\quad(\mathrm{GHz})\end{array}$ & Array & $\begin{array}{l}\text { Observing } \\
\text { Time } \\
\text { (s) }\end{array}$ & $\begin{array}{l}\text { Resolution } \\
\quad(\operatorname{arcsec})\end{array}$ & Comment \\
\hline \multirow[t]{7}{*}{$3 \mathrm{C} 263 \ldots \ldots$} & AH737 & 2001 Jan 3 & 14.94 & A & 4390 & $0.14 \times 0.10$ & Our observations \\
\hline & AP380 & 2000 Apr 27 & 4.86 & $\mathrm{C}$ & 1500 & $4.67 \times 3.31$ & Map supplied by L. M. Mullin \\
\hline & AL270 & 1992 Oct 31 & 1.44 & A & 2930 & $1.51 \times 1.12$ & Archival $u v$ data \\
\hline & AW249 & 1991 Aug 31 & 8.26 & A & 1150 & $0.28 \times 0.19$ & Archival $u v$ data \\
\hline & AW249 & 1991 Nov 13 & 8.26 & B & 1160 & $0.87 \times 0.69$ & Archival $u v$ data \\
\hline & AB454 & 1987 Jul 11 & 4.87 & A & 23940 & 0.36 & Map supplied by R. A. Laing (1) \\
\hline & $\mathrm{AB} 454$ & 1987 Dec 6 & 4.87 & $\mathrm{~B}$ & 14300 & 1.00 & Map supplied by R. A. Laing (1) \\
\hline \multirow[t]{6}{*}{$3 \mathrm{C} 330 \ldots \ldots$} & AP331 & 1996 Dec 15 & 8.47 & A & 5050 & 0.30 & Map supplied by J. M. Riley (2) \\
\hline & AP331 & 1997 Apr 13 & 8.47 & B & 2920 & & Map supplied by J. M. Riley (2) \\
\hline & AL200 & 1989 Sep 19 & 8.41 & $\mathrm{C}$ & 10170 & 2.50 & Map supplied by J. M. Riley (2) \\
\hline & AL200 & 1989 Nov 24 & 8.41 & $\mathrm{D}$ & 2570 & & Map supplied by J. M. Riley (2) \\
\hline & AM213 & 1987 Aug 17 & 1.49 & A & 2050 & $1.52 \times 1.18$ & Archival $u v$ data \\
\hline & AA114 & 1991 Jan 24 & 14.94 & $\mathrm{C}$ & 2850 & $1.49 \times 1.13$ & Archival $u v$ data \\
\hline \multirow[t]{8}{*}{$3 \mathrm{C} 351 \ldots \ldots}$. & LAIN & 1982 Mar 11 & 1.42 & A & 3190 & 1.85 & 3CRR Atlas ${ }^{\mathrm{a}}$ (3) \\
\hline & AL146 & 1987 Nov 25 & 1.47 & B & 3370 & & 3CRR Atlas ${ }^{\mathrm{a}}$ (3) \\
\hline & AW249 & 1991 Jun 30 & 8.06 & A & 2360 & $0.33 \times 0.23$ & Archival $u v$ data \\
\hline & AW249 & 1991 Nov 13 & 8.06 & B & 2360 & & Archival $u v$ data \\
\hline & AW249 & 1991 Jan 24 & 8.06 & $\mathrm{C}$ & 2350 & 3.0 & Map supplied by J. M. Riley (2) \\
\hline & AP331 & 1998 Jan 24 & 8.47 & $\mathrm{D}$ & 810 & & Map supplied by J. M. Riley (2) \\
\hline & AL43 & 1983 Мay 6 & 14.96 & $\mathrm{C}$ & 3500 & $1.36 \times 1.10$ & Archival $u v$ data \\
\hline & LAIN & 1982 May 30 & 14.96 & A & 1150 & $0.15 \times 0.10$ & Archical $u v$ data \\
\hline
\end{tabular}

NotE.- The resolutions quoted are the FWHM of the restoring circular Gaussian, if a circular beam was used, or otherwise the major and minor FWHM of the restoring elliptical Gaussian: as maps were made from combinations of different observations in several cases, not all individual observations have corresponding resolutions quoted. Observing times are taken from the VLA archive and do not include the effects of any required data flagging.

a The 3CRR Atlas can be found online at http://www.jb.man.ac.uk/atlas.

References.- (1) Bridle et al. 1994; (2) Gilbert et al. 2002; (3) Leahy \& Perley 1991.

the CIAO script psextract ${ }^{1}$ and analyzed with XSPEC. Particular care was taken in the case of the lobe extractions to ensure that the background regions used were at similar or identical distances from the nucleus (Fig. 2); this minimizes systematic effects due to the radially symmetrical pointspread function (PSF) or any radially symmetrical cluster emission. In all cases, spectra were binned such that each bin contained $\gtrsim 20$ net counts. Fits were carried out in the 0.4-7.0 keV energy band. The models used for each source are discussed in detail in the following sections; the results of fitting these models are tabulated for each source in Table 8. Errors quoted in the table or in the text correspond

${ }^{1}$ This script extracts spectra from regions of the events file and a chosen background and generates appropriate response files. See http:// cxc.harvard.edu/ciao/ahelp/psextract.html.

TABLE 5

BIMA OBSERVATIONS

\begin{tabular}{|c|c|c|c|}
\hline Source & Date & $\begin{array}{l}\text { Duration } \\
\text { (hr) }\end{array}$ & $\begin{array}{c}\text { On-Source } \\
\text { Time } \\
\text { (hr) }\end{array}$ \\
\hline 3С $263 \ldots \ldots \ldots \ldots$ & 2002 Feb 3 & 8.0 & 4.0 \\
\hline 3C $330 \ldots \ldots \ldots \ldots$ & 2002 Feb 8 & 4.8 & 2.5 \\
\hline 3C $351 \ldots \ldots \ldots \ldots . . . .$. & 2002 Feb 10 & 7.5 & 3.9 \\
\hline
\end{tabular}

NotE.-The duration given is the entire length of the run, including slewing and calibration: this indicates the $u v$ coverage obtained. The on-source time is the time spent observing the target, and indicates the sensitivity of the observations. to the $1 \sigma$ uncertainty for one interesting parameter, unless otherwise stated.

\subsection{Intracluster Medium Emission}

Our deep Chandra observations also allow us to search for extended, cluster-related X-ray emission. Extended emission, on scales larger than the radio lobes, was visible by eye in the observations of 3C 263 and 3C 330. To characterize this extended emission we masked out the readout streak and the nonnuclear emission (hot spots and lobes), using conservatively large masking regions that effectively covered the whole extended radio source in each case, and then generated a radial profile for each object. We chose to represent the extended emission with isothermal $\beta$ models (Cavaliere \& Fusco-Femiano 1978), which give rise to an angular distribution of counts on the sky of the form

$$
b_{\mathbf{X}}(\theta)=b_{0}\left(1+\frac{\theta^{2}}{\theta_{c}^{2}}\right)^{(1 / 2)-3 \beta},
$$

where $b_{\mathrm{X}}$ is the count density (in counts $\operatorname{arcsec}^{-2}$ ) as a function of off-source angle $\theta, b_{0}$ is the central count density, and $\theta_{c}$ and $\beta$ parameterize the angular scale and shape of the extended emission. Modeling the background-subtracted radial profiles as a combination of a delta function and a $\beta$ model, both convolved with the PSF, we then carried out least-squares fits using a grid of $\beta$ and $\theta_{c}$ values to find out whether extended emission was required and to determine the best-fitting $\beta$ model parameters.

To do this we required an analytical model of the PSF. Worrall et al. (2001a, 2001b) discussed the fitting of 
TABLE 6

HST WFPC2 OBSERVATIONS USED In The ANALysis

\begin{tabular}{|c|c|c|c|c|c|}
\hline Source & ID & Date & Filter & $\begin{array}{l}\text { Duration } \\
\text { (s) }\end{array}$ & $\begin{array}{c}\text { Observatory } \\
\text { Roll Angle }\end{array}$ \\
\hline $3 \mathrm{C} 263 \ldots$ & U2SE0201 & 1996 Feb 18 & F675W & 1000 & 28.68 \\
\hline 3C $330 \ldots \ldots \ldots \ldots$ & U3A14X01 & 1996 Jun 03 & F555W & 600 & 333.46 \\
\hline 3C $351 \ldots \ldots \ldots \ldots . . . . .$. & U2X30601 & 1995 Nov 30 & F702W & 2400 & 54.73 \\
\hline
\end{tabular}

NotE.-The satellite roll angle quoted is the measured position angle on the sky of the $H S T$ 's V3 axis, north through east. The orientation of the science images on the sky (the position angle of the detector $y$ axis) is the sum of this angle and the angle between the V 3 axis and the detector $y$-axis, $\sim 224$. 6 for these observations.

analytical forms to data from the CIAO PSF library, and our approach is similar. Our data are derived from the CIAO mkpsf command, ${ }^{2}$ energy-weighted to reflect the approximate energy distribution of the observed data in the extraction radius, smoothed with a small Gaussian to

2 This command generates images of the Chandra point-spread function at a given energy from the PSF library, which is itself derived from ray-tracing simulations of the X-ray mirror assembly. See http:// cxc.harvard.edu/ciao/ahelp/mkpsf.html and http://cxc.harvard.edu/ caldb/cxcpsflib.manual.ps for more information. simulate the effects of pixelation and aspect uncertainties, and then fitted with a suitable function of radius. The forms used by Worrall et al., although a good fit to the inner regions of the PSF and so adequate for the purposes used in their papers, do not represent the wings found at radial distances of tens of arcseconds, and these are important in the case of $3 \mathrm{C} 263$ and $3 \mathrm{C} 351$, where the emission is dominated by the bright central point source out to large radii. Accordingly, we fitted a functional form consisting of two Gaussians, two exponentials, and a power-law component, which we found empirically to be a good fit out to large distances,

TABLE 7

Spatial Regions Used in Extraction

\begin{tabular}{|c|c|c|c|c|c|}
\hline \multirow[b]{2}{*}{ SOURCE } & \multirow[b]{2}{*}{ Component } & \multicolumn{2}{|c|}{ Central Position } & \multirow[b]{2}{*}{ GeOMEtry } & \multirow[b]{2}{*}{ Number } \\
\hline & & R.A. & Decl. & & \\
\hline \multirow[t]{10}{*}{$3 \mathrm{C} 263 \ldots \ldots \ldots$} & Core & 113957.025 & +654749.36 & Circle, $r=5$ pixels & 1 \\
\hline & Southeast hot spot (IJK) & 113959.457 & +654743.59 & Circle, $r=2.9$ pixels & 2 \\
\hline & Southeast hot spot (K) & 113959.492 & +654743.47 & Circle, $r=1.4$ pixels & \\
\hline & Southeast hot spot background & 114000.361 & +654757.06 & Circle, $r=13.4$ pixels & 3 \\
\hline & Northwest hot spot (limit region) & 113952.857 & +654759.57 & Circle, $r=12.6$ pixels & 4 \\
\hline & Northwest lobe & 113952.857 & +654759.57 & Circle, $r=12.6$ pixels & 4 \\
\hline & NW lobe background & 113948.821 & +654801.59 & Circle, $r=31.6$ pixels & 5 \\
\hline & Jet (limit region) & 113959.090 & +654749.30 & Box, $22.6 \times 12.6$ pixels, $90^{\circ}$ & 6 \\
\hline & Southeast lobe & 113959.090 & +654749.30 & Box, $22.6 \times 12.6$ pixels, $90^{\circ}$ & 6 \\
\hline & Southeast lobe background & 113955.030 & +654749.16 & Box, $22.6 \times 12.6$ pixels, $90^{\circ}$ & 7 \\
\hline \multirow[t]{10}{*}{$3 \mathrm{C} 330 \ldots \ldots \ldots$} & Core & 160934.896 & +655637.69 & Circle, $r=7.3$ pixels & 1 \\
\hline & Northeast hot spot & 160939.155 & +655652.79 & Circle, $r=7.3$ pixels & 2 \\
\hline & Southwest hot spot & 160930.255 & +655623.07 & Circle, $r=3.25$ pixels & 3 \\
\hline & hot spot/core background & 160941.548 & +655613.74 & Circle, $r=65.5$ pixels & 4 \\
\hline & Northeast lobe & 160937.433 & +655647.29 & Box, $31.1 \times 16.0$ pixels, 59.5 & 5 \\
\hline & Northeast lobe background & 160936.608 & +655655.90 & Box, $31.1 \times 16.0$ pixels, 59.5 & 6 \\
\hline & & 160938.198 & +655639.30 & Box, $31.1 \times 16.0$ pixels, 59.5 & 7 \\
\hline & Southwest lobe & 160932.083 & +655628.83 & Box, $31.1 \times 16.0$ pixels, 66.5 & 8 \\
\hline & Southwest lobe background & 160931.419 & +655637.44 & Box, $31.1 \times 16.0$ pixels, 66.5 & 9 \\
\hline & & 160932.687 & +655619.49 & Box, $31.1 \times 16.0$ pixels, 66.5 & 10 \\
\hline \multirow[t]{9}{*}{$3 \mathrm{C} 351 \ldots \ldots \ldots$} & Core & 170441.364 & +604430.46 & Circle, $r=12.8$ pixels & 1 \\
\hline & Core background & 170441.364 & +604430.46 & Annulus, $r=12.8 \ldots 25$ pixels & \\
\hline & North hot spot (J) & 170443.781 & +604448.20 & Circle, $r=5$ pixels & 2 \\
\hline & North hot spot (L) & 170443.479 & +604452.88 & Circle, $r=5$ pixels & 3 \\
\hline & North hot spots background & 170445.525 & +604433.19 & Circle, $r=22.5$ pixels & 4 \\
\hline & South hot spot (limit region) & 170439.754 & +604404.91 & Circle, $r=16$ pixels & \\
\hline & North lobe & 170440.760 & +604455.09 & Circle, $r=29$ pixels & 5 \\
\hline & South lobe & 170439.888 & +604407.37 & Circle, $r=29$ pixels & 6 \\
\hline & Lobe background & 170443.646 & +604409.83 & Circle, $r=29$ pixels & 7 \\
\hline
\end{tabular}

Note.- Units of right ascension are hours, minutes, and seconds, and units of declination are degrees, arcminutes, and arcseconds. Positions quoted are corrected to the frame defined by the radio observations, as discussed in the text. One Chandra pixel is 0 " 492. "Limit regions" are the regions used to determine a background count rate for an upper limit on a compact component; smaller detection regions are then used to derive the upper limit, as discussed in the text. Box regions are given as long axis $\times$ short axis, position angle of long axis (defined north through east). Numbers refer to the regions shown in Fig. 2. 

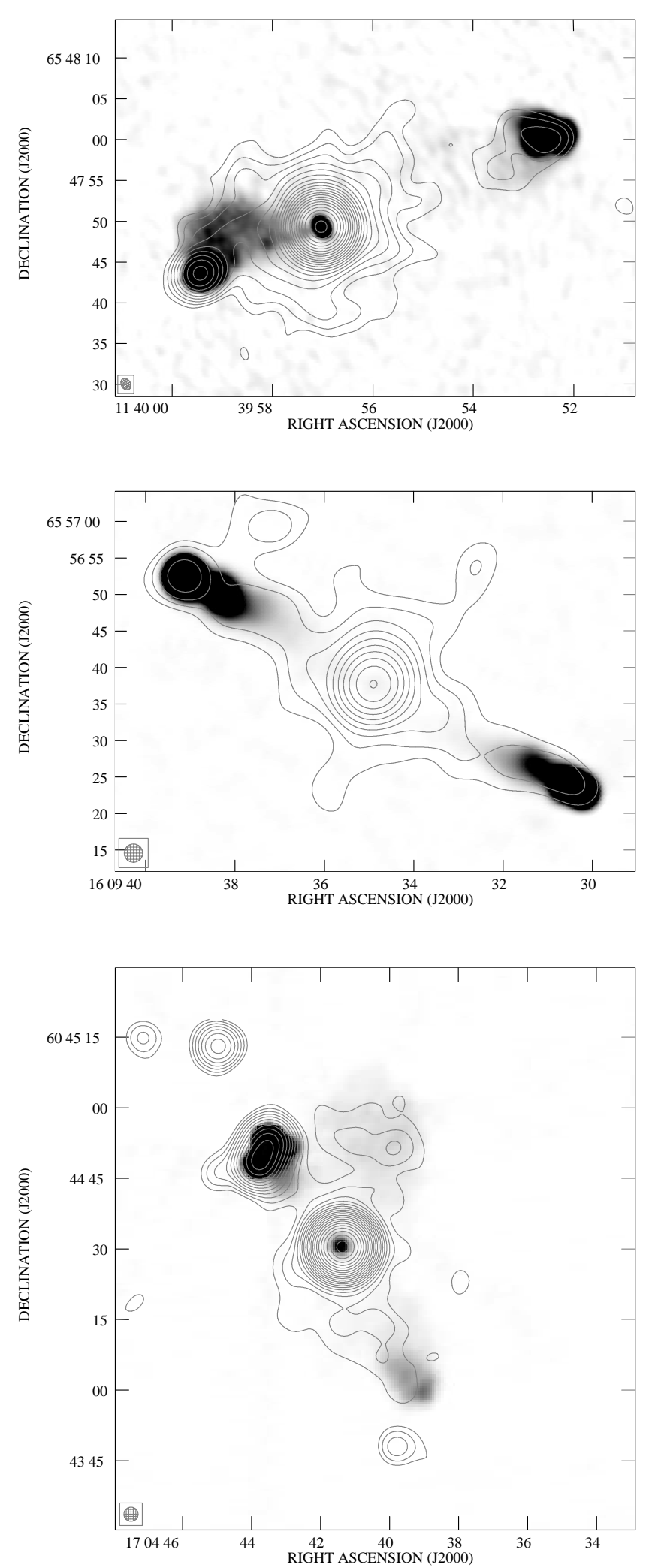

FIG. 1.-Three X-ray sources. From top to bottom: 3C 263, 3C 330, and 3C 351. The contours show the X-ray data, smoothed with a Gaussian of FWHM 3".5 (for 3C 263) and 6" (for the other two sources): the contour intervals are logarithmic in steps of $\sqrt{2}$, and the lowest contour in each figure is the $3 \sigma$ level as defined by Hardcastle (2001). The objects to the northeast and south of $3 \mathrm{C} 351$ are background point sources. The gray scales show radio maps. The map of $3 \mathrm{C} 263$ is a $1.4 \mathrm{GHz}$ VLA image with 1 . $5 \times 1$ ". 1 resolution; black is $10 \mathrm{mJy}$ beam $^{-1}$. The $3 \mathrm{C} 330$ and $3 \mathrm{C} 351 \mathrm{maps}$ are $8.4 \mathrm{GHz}$ VLA images, from Gilbert et al. (2002), with 3" resolution; black is $10 \mathrm{mJy}^{\text {beam }}{ }^{-1}$.
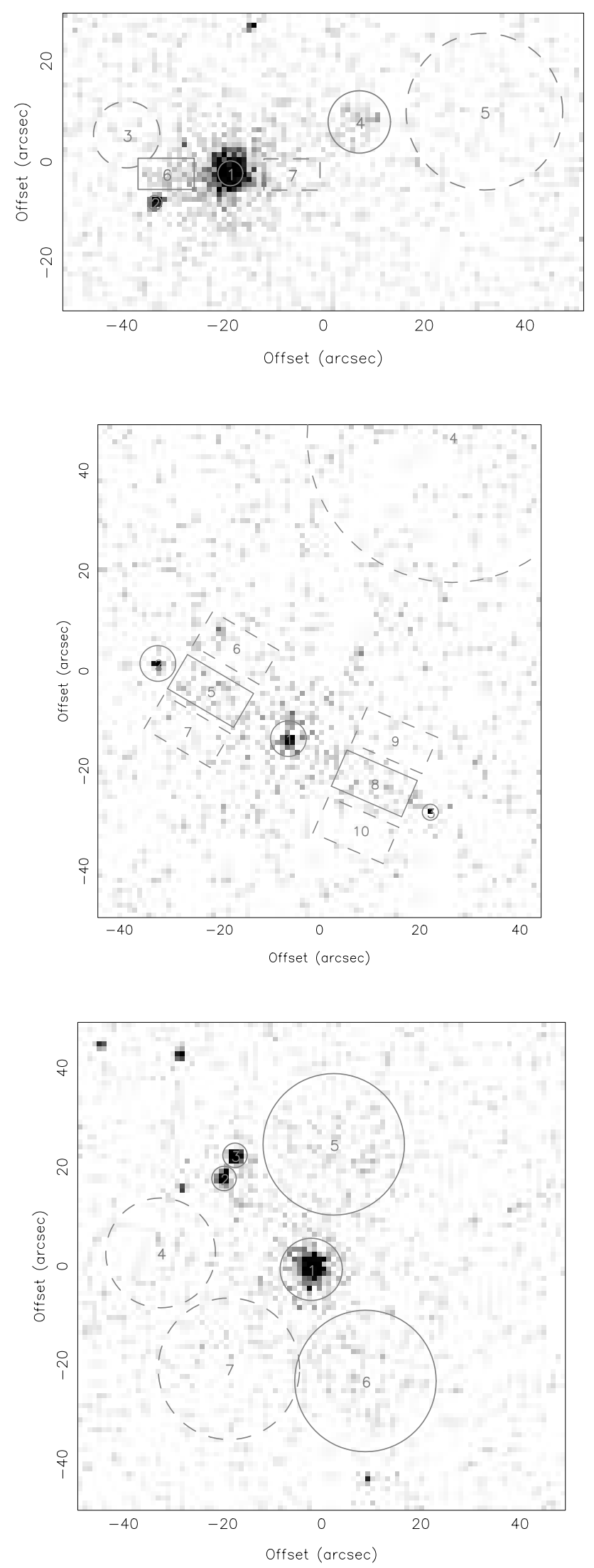

FIG. 2.-Extraction regions used in the three radio sources. From top to bottom: 3C 263, 3C 330, and 3C 351. Each image shows the $0.4-7 \mathrm{keV}$ events binned by a factor of 2 (i.e., to 0 ".984 pixels); black is 8,5 , and 8 counts per bin, respectively. Solid lines show source regions and dashed lines background regions. Numbers refer to the regions listed in Table 7. For clarity, a few regions have been omitted. 


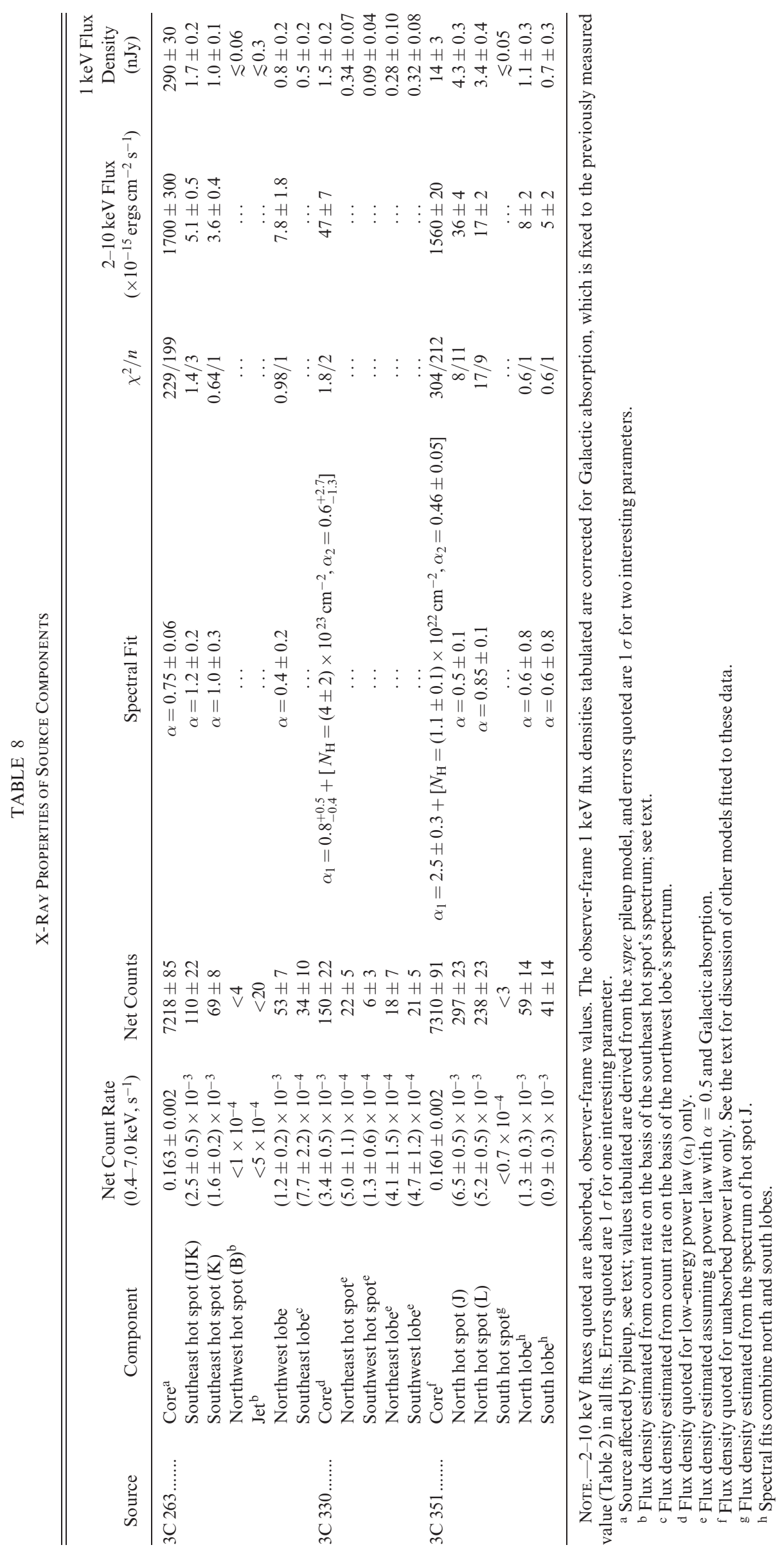


TABLE 9

Radio Flux Densities for Source Components

\begin{tabular}{|c|c|c|c|c|}
\hline Source & Component & Model & $\begin{array}{c}\text { Frequency } \\
\quad(\mathrm{GHz})\end{array}$ & $\begin{array}{l}\text { Flux Density } \\
(\mathrm{mJy})\end{array}$ \\
\hline \multirow[t]{12}{*}{$3 C 263 \ldots \ldots}$. & \multirow[t]{5}{*}{ Hot spot K } & \multirow[t]{5}{*}{ Sphere, $r=0 " 139^{\mathrm{a}}$} & 1.4 & 1670 \\
\hline & & & 4.8 & 591 \\
\hline & & & 8.3 & 303 \\
\hline & & & 14.9 & 184 \\
\hline & & & 84.9 & 54 \\
\hline & \multirow[t]{3}{*}{ Hot spot B } & \multirow{3}{*}{ Sphere, $r=0$ ". 18} & 4.8 & 23 \\
\hline & & & 8.3 & 16 \\
\hline & & & 14.9 & 9 \\
\hline & \multirow[t]{2}{*}{ South lobe (X-ray region) } & \multirow[t]{2}{*}{ Cube, 9 ". $5 \times 6$ ". $3 \times 9$ ". 3} & 1.4 & 206 \\
\hline & & & 4.9 & 45 \\
\hline & \multirow[t]{2}{*}{ North lobe } & \multirow{2}{*}{ Sphere, $r=6^{\prime \prime}$} & 1.4 & 550 \\
\hline & & & 4.9 & 188 \\
\hline \multirow[t]{11}{*}{$3 \mathrm{C} 330 \ldots \ldots$} & \multirow[t]{4}{*}{ Northeast hot spot } & \multirow[t]{4}{*}{ Cylinder, 2 ". $3 \times 0$ ".64 } & 1.5 & 3830 \\
\hline & & & 8.4 & 755 \\
\hline & & & 14.9 & 376 \\
\hline & & & 84.9 & 25 \\
\hline & Southwest hot spot & Sphere, $r=0 "$. 19 & 8.4 & 0.77 \\
\hline & \multirow[t]{3}{*}{ Northeast lobe (X-ray region) } & \multirow[t]{3}{*}{ Cylinder, $15^{\prime \prime} \times 2$ ".6 } & 1.5 & 737 \\
\hline & & & 8.4 & 161 \\
\hline & & & 14.9 & 63 \\
\hline & \multirow[t]{3}{*}{ Southwest lobe (X-ray region) } & \multirow[t]{3}{*}{ Cylinder, $15^{\prime \prime} \times 2$ ".6 } & 1.5 & 768 \\
\hline & & & 8.4 & 135 \\
\hline & & & 14.9 & 55 \\
\hline \multirow{13}{*}{$3 \mathrm{C} 351 \ldots \ldots$} & \multirow{4}{*}{ Hot spot $\mathbf{J}$} & \multirow{4}{*}{ Sphere, $r=0 " 16$} & 1.4 & 530 \\
\hline & & & 8.4 & 130 \\
\hline & & & 15.0 & 88 \\
\hline & & & 84.9 & 17 \\
\hline & \multirow[t]{4}{*}{ Hot spot L } & \multirow[t]{4}{*}{ Sphere, $r=0 . " 8$} & 1.4 & 1300 \\
\hline & & & 8.4 & 316 \\
\hline & & & 15.0 & 201 \\
\hline & & & 84.9 & 28 \\
\hline & Hot spot A & Two spheres, $r=0$ ". 35 & 8.4 & 3 \\
\hline & \multirow[t]{2}{*}{ North lobe (X-ray region) } & \multirow[t]{2}{*}{ Sphere, $r=10 " .5$} & 1.4 & 283 \\
\hline & & & 8.3 & 43 \\
\hline & \multirow[t]{2}{*}{ South lobe (X-ray region) } & \multirow[t]{2}{*}{ Cylinder, $28^{\prime \prime} \times 6 . .5$} & 1.4 & 248 \\
\hline & & & 8.3 & 46 \\
\hline
\end{tabular}

Note.-Errors on the hot spot flux densities are due primarily to flux calibration uncertainties, nominally $2 \%$ for the VLA data and $10 \%$ for the $85 \mathrm{GHz}$ BIMA data, as discussed in $\S 2$.

a See the text for a discussion of the more detailed spatial models applied to this hot spot in the inverse Compton calculation.

to the PSF $a(\theta)$ :

$$
\begin{aligned}
a(\theta)= & a_{1} \exp \left(-\frac{\theta^{2}}{2 s_{1}^{2}}\right)+a_{2} \exp \left(-\frac{\theta^{2}}{2 s_{2}^{2}}\right)+a_{3} \exp \left(-\frac{\theta}{s_{3}}\right) \\
& +a_{4} \exp \left(-\frac{\theta}{s_{4}}\right)+a_{5}\left(1+\frac{\theta}{\theta_{n}}\right)^{s_{5}},
\end{aligned}
$$

where $a_{1}, \ldots, a_{5}, s_{1}, \ldots, s_{5}$ are parameters of the fit and $\theta_{n}$ is a fixed normalizing value. In practice, the power-law index $s_{5}$ was determined using the lowest resolution PSF libraries and then fixed, while the other parameters were determined from higher resolution data. In using a power-law model for the PSF wings, we have followed other work on characterizing the large-radius $\mathrm{PSF},{ }^{3}$ and we have obtained similar power-law indices, $s_{5} \approx 2.0$, in all the fits we have carried out.

\footnotetext{
${ }^{3}$ See, e.g., documents at http://asc.harvard.edu/cal/Hrma/hrma/psf.
}

\subsection{Inverse Compton Modeling}

To compare the observed X-ray emission from the lobes and hot spots to the predictions of inverse Compton models it is necessary to know the spatial and spectral structure of these components in the radio and optical in as much detail as possible. The available data in these bands are discussed in the following sections, but in each case we extracted and tabulated radio flux densities (Table 9) and derived radiobased models for the hot spots and lobes. Where these components were well resolved, we measured their sizes directly from radio maps; where they were compact, as was the case for some of the hot spots, we characterized the component size by fitting a model of the emission from an optically thin homogeneous sphere, convolved with the restoring Gaussian, to the high-resolution radio maps.

We then used a computer code to predict inverse Compton flux density as a function of magnetic field strength. Two inverse Compton codes were available to us. One, described by Hardcastle, Birkinshaw, \& Worrall (1998), 
treats all inverse Compton sources as homogeneous spheres; this allows us to neglect the anisotropy of inverse Compton emission and so gives a quick and simple calculation. Our other code is based on the results of Brunetti (2000). By incorporating Brunetti's formulation of anisotropic inverse Compton scattering, this code allows us to take account of the spatial and spectral structure of the resolved hot spots. To model spatial structure, the hot spots are placed on a fine three-dimensional grid and the emissivity resulting from the illumination of every cell by every other cell is calculated (making small corrections for self-illumination and nearest neighbor effects). To do this in full generality is computationally expensive, since it requires numerical integration of equations (A1)-(A5) of Brunetti (2000) over the electron distribution and incoming photon distribution for each cell in an $O\left(n^{6}\right)$ algorithm, where $n$ is the number of cells in one dimension of the three-dimensional grid. We solve this problem by allowing only a small number of distinct electron spectra and magnetic fields in our grid. This means that the task of calculating the illumination of a region of electrons of a given spectral shape by a given photon distribution reduces to one of tabulating the integral of Brunetti's results for a suitably sampled subset of the possible values of the geometry parameter $k_{3}$. Since the dependence on the normalization of the electron energy spectrum and on the incoming photon flux is linear, the $O\left(n^{6}\right)$ part of the algorithm then just involves an interpolation and multiplication. For homogeneous spheres, or in the case of cosmic microwave background (CMB) inverse Compton scattering $(\mathrm{CMB} / \mathrm{IC})$ where anisotropy is not an issue, the results of our two codes agree to within a few percent, and so in the results presented below we use whichever code is most appropriate for a particular situation.

The synchrotron spectra of the hot spots and lobes studied in this work are not as well studied as those of earlier targets, so some assumptions are necessary in modeling them. Our basic model is a broken power-law electron energy spectrum, such that

$$
N(\gamma)= \begin{cases}0 & \gamma<\gamma_{\text {min }}, \\ N_{0} \gamma^{-p} & \gamma_{\text {min }}<\gamma<\gamma_{\text {break }}, \\ N_{0}\left(\gamma_{\text {break }}^{d}\right) \gamma^{-(p+d)} & \gamma_{\text {break }}<\gamma<\gamma_{\text {max }}, \\ 0 & \gamma>\gamma_{\text {max }},\end{cases}
$$

where $N(\gamma) d \gamma$ gives the number density of electrons with Lorentz factors between $\gamma$ and $\gamma+d \gamma$. We choose this model because synchrotron theory tells us that it is a good approximation to the expected situation in hot spots, where particle acceleration and synchrotron losses are competing; for $d=1$ it approximates the standard "continuous injection" model, which applies to a region containing the acceleration site and a downstream region of synchrotron radiation loss (e.g., Heavens \& Meisenheimer 1987). Theoretical prejudice (based on models of shock acceleration) also suggests a value $p=2$ for the low-energy power-law index (the "injection index"). This electron energy spectrum gives rise to a synchrotron spectrum whose spectral index $\alpha$ is flat or inverted $\left(\alpha=-\frac{1}{3}\right)$ around and below some frequency $\nu_{\text {turn }}$, has a value 0.5 up to around some break frequency $\nu_{\text {break }}$, and then steepens to a value of 1.0 before an exponential cutoff around some frequency $\nu_{\text {cutoff }}$. Such models have been successfully fitted to a number of wellstudied hot spots (e.g., Meisenheimer et al. 1989; Looney \&
Hardcastle 2000). It is well known that the synchrotron turnover, break and cutoff are not sharp; for typical parameters of a radio source they occupy a decade or more in frequency. It is for this reason that we prefer to work with the well-defined and physically interesting quantities $\gamma_{\min }$, $\gamma_{\text {break }}, \gamma_{\max }$, whose only disadvantage is that they must be specified together with a value of the magnetic field strength $B$. We typically work with the equipartition magnetic field strength, $B_{\text {eq }}$, which is given by

$$
m_{\mathrm{e}} c^{2} \int_{\gamma_{\min }}^{\gamma_{\max }} \gamma N(\gamma) \mathrm{d} \gamma=B_{\mathrm{eq}}^{2} / 2 \mu_{0}
$$

(where we have assumed that equipartition is between the radiating electrons and the magnetic field only, and where SI units are used, with $\mu_{0}$ being the permeability of free space).

If there is evidence for spectral steepening to $\alpha>1$ in the synchrotron spectrum, $\gamma_{\max }$ is constrained. We find that $\gamma_{\text {break }}$ is often in the observed radio region, so that it is relatively easy to constrain. We therefore estimate $\gamma_{\max }$ and $\gamma_{\text {break }}$ using least-squares fitting to the radio and optical data. (Typically $\gamma_{\max }$ lies above the radio region, in which case its value has little effect on our inverse Compton calculations.) However, $\gamma_{\min }$ can be directly constrained only by observations of a low-frequency turnover in sources that are not synchrotron selfabsorbed (Carilli et al. 1991; Hardcastle et al. 2001a; Hardcastle 2001) or by observations of optical inverse Compton emission (Hardcastle 2001), and neither of these methods has yet been applied to the hot spots or lobes of our sources. Our low-frequency data on the hot spots typically constrain $\gamma_{\min } \lesssim 1000$ for equipartition fields, but values of $\gamma_{\min }$ between 400 and 1000 have been reported for other objects (Hardcastle 2001 and references therein). In addition, it is possible, as argued by Brunetti et al. (2002a), that the electron spectrum at low energies should be modified by the effects of adiabatic expansion out of the hot spot. Fortunately, the values of $\gamma_{\min }$ and the low-energy electron spectral shape have only a small effect on the inverse Compton calculation, because the broken synchrotron spectrum produces few high-energy photons that can be scattered into the X-ray by such low-energy electrons. Unless otherwise stated we assume $\gamma_{\min }=1000$.

\section{4. $3 \mathrm{C} 263$}

\subsection{Introduction}

$3 \mathrm{C} 263$ is a $z=0.66$ quasar. In the radio, the best maps are those of Bridle et al. (1994, hereafter B94), who show it to have a one-sided jet that points toward a bright, compact hot spot in the southeast lobe. The source lies in an optically rich region, and some nearby objects' associations with the quasar has been spectroscopically confirmed; HST observations show several close small companions. Deep X-ray images were made with ROSAT by Hall et al. (1995) in an attempt to locate the X-ray emission from the host cluster, but no significant extended emission on cluster scales was found by them or by Hardcastle \& Worrall (1999), who reanalyzed their data. The ROSAT data were dominated by the quasar's bright, variable nuclear X-ray component. 


\subsection{Core}

The bright X-ray nucleus of 3C 263 suffers from pileup in our full-frame observation. The piled-up count rate over the full Chandra band is $0.190 \pm 0.002 \mathrm{~s}^{-1}$, so we would expect a pileup fraction of about $20 \%$. When the core is fitted with a power-law spectrum without making any correction for pileup, there is no sign of any excess absorption over the (small) Galactic value, but the best-fit power law is very flat $(\alpha \approx 0.1)$ and there are substantial residuals around $2 \mathrm{keV}$, which are both characteristic of piled-up spectra. Using the pileup model in XSPEC, which implements the work of Davis (2001), we find a good fit with a range of steeper power laws. Following Davis (2001), we fix the grade correction parameter $\bar{g}_{0}$ to 1 and allow only the grade morphing parameter to vary. ${ }^{4}$ We find the best fit (Table 8) to have $\alpha=0.75 \pm 0.06$ (since the slope and normalization are strongly correlated in this model, the error quoted is $1 \sigma$ for two interesting parameters), which is in reasonable agreement with previous observations; for example, in the compilation of Malaguti, Bassani, \& Caroli (1994) $\alpha$ values between 0.7 and 1.0 are reported. The pileup-corrected 1 $\mathrm{keV}$ flux density is substantially lower than the value we estimated (based on a similar spectral assumption) from the ROSAT HRI data (Hardcastle \& Worrall 1999), suggesting variability by a factor of $\sim 2$ on a timescale of years (the ROSAT data were taken in 1993). This is consistent with the observations of Hall et al. (1995), who saw variability by a factor of 1.5 in 18 months.

Using the default Chandra astrometry, the core centroid is at $11^{\mathrm{h}} 39^{\mathrm{m}} 57^{\mathrm{s}} .170,+65^{\circ} 47^{\prime} 49^{\prime \prime} .22$, while our best position for the radio core, using our $15 \mathrm{GHz}$ VLA data, is $11^{\mathrm{h}} 39^{\mathrm{m}} 57^{\mathrm{s}} .025,+65^{\circ} 47^{\prime} 49^{\prime \prime} \cdot 36$, which is within 0.05 of the position quoted by B94. The Chandra position is thus offset by approximately 0 ." 89 in R.A. and 0 ". 14 in declination from the radio data, which is consistent with known Chandra astrometric uncertainties. In our spatial analysis we shift the Chandra data so that the core positions match and align other radio and $H S T$ data with our best core position.

\subsection{Hot Spots}

The bright southeast hot spot of 3C 263 is detected in the Chandra observation; it corresponds to a faint feature seen in the ROSAT HRI images by Hall et al. (1995), which they describe as a "clump" of X-ray emission. As Figure 3 shows, we detect emission with Chandra not just from the compact component $\mathrm{K}$ (we use the notation of B94) but also the faint tail $\mathbf{J}$ (which may trace the incoming jet) and the trailing plateau of emission I. The X-ray/radio ratio is considerably higher in $J$ (by a factor of $\sim 4$ ) than in the other components. The high-resolution images show that the $\mathrm{X}$-ray centroid of the compact component $\mathrm{K}$ is slightly to the north of the peak of the radio emission. The offset is about 0 "!3, or $2 \mathrm{kpc}$. The angular displacement about the pointing center, 1.1 , is too large by an order of magnitude to be due to Chandra roll uncertainties, which are typically at most 0.1 (T. Aldcroft 2002, private communication); we conclude that the offset is real. We extracted spectra from the entire complex IJK and from the compact hot spot K. The net count rate from the IJK complex is consistent,

\footnotetext{
${ }^{4}$ The model is prone to getting stuck in local minima, and so we explored parameter space using steppar.
}

within the errors, with the count rate estimated by Hall et al. for the "clump" in their ROSAT data, converted to an equivalent Chandra count rate.

A faint optical counterpart to $\mathrm{K}$ is detected in the $1000 \mathrm{~s}$ F675W HST observation of 3C 263 (Fig. 4). After aligning the radio core with the peak of the optical quasar emission, the peak optical and radio positions of the hot spot agree to within 0 ". 1. (HST roll uncertainties are usually no worse than 0.05 , corresponding to an error at this distance from the pointing center of 0. ". 01 .) We estimate a flux density for this component at $4.5 \times 10^{14} \mathrm{~Hz}$ of $0.8 \pm 0.4 \mu \mathrm{Jy}$, with the large error arising because of uncertainties in background estimation.

As Figure 4 shows, the hot spot has considerable spatial structure in the radio. Even the compact component $\mathrm{K}$ is shown by our $15 \mathrm{GHz}$ images to have several subcomponents; a bright central component K1, an extended halo K2, and a weak leading compact component $\mathrm{K} 3$. The radio spectrum of hot spot K (Fig. 5), which we obtain by Gaussian fitting to the $1.4 \mathrm{GHz}$ map and by integration of matched regions on the higher resolution 4.8, 8.3, and 15 $\mathrm{GHz}$ maps, also shows signs of multiple structure in that the spectrum steepens between 4.8 and $8.3 \mathrm{GHz}$ but then flattens again between 8.3 and $15 \mathrm{GHz}$. The $8.4-15 \mathrm{GHz}$ spectral index maps show that the compact central component $\mathrm{K} 1$ has a flatter two-point radio spectrum, $\alpha \approx 0.8$, while the surrounding material is steeper-spectrum, $\alpha \approx 1.0$. Correcting for background contamination, $\alpha_{\mathrm{K} 1} \approx 0.7$ between 8.3 and $15 \mathrm{GHz}$, which is consistent with the observed 15 $85 \mathrm{GHz}$ spectral index. We can crudely model hot spot $\mathrm{K}$ as being a superposition of two of our standard spectra with different break frequencies, with the K1 component breaking above $85 \mathrm{GHz}\left(\gamma_{\text {break }} \approx 2 \times 10^{4}\right.$, assuming equipartition magnetic fields), while the $\mathrm{K} 2 / \mathrm{K} 3$ components break at 1.4 $\mathrm{GHz}$ or lower $\left(\gamma_{\text {break }} \approx 1000\right)$. In order for the K1 component not to overproduce the observed optical emission, assumed to be synchrotron emission, its spectrum must then steepen further at high energies; we choose to model this with a high-energy cutoff in the electron energy spectrum, $\gamma_{\max }=5 \times 10^{5}$. For simplicity, we assign the extended component the same high-energy cutoff, which makes its contribution to the observed optical emission negligible. We modeled $\mathrm{K} 1$ as a sphere of radius 0 ". 15 and $\mathrm{K} 2$ as a sphere of radius 0.39 , based on the $15 \mathrm{GHz}$ map. This synchrotron model is plotted in Figure 5.

Three important unknown factors remain: the geometry of $\mathrm{K} 1 / \mathrm{K} 2$ (does the compact component $\mathrm{K} 1$ lie inside or outside, in front of or behind, the more extended component K2?), the spatial electron distribution in the two spheres (uniform or centrally peaked? - the radio data are not good enough to say), and the low-energy cutoff in the synchrotron spectrum. The $1.4 \mathrm{GHz}$ observations suggest a low-energy cutoff $\gamma_{\min } \lesssim 1000$; a higher cutoff would mean we would start to observe spectral flattening at this frequency. We carried out our inverse Compton modeling with a range of possible geometries, spatial electron distributions, and $\gamma_{\min }$ values in order to assess the effect of these differences. We find that the most significant effect is given by a more centrally peaked distribution of electrons; $\gamma_{\text {min }}$ has little effect, and only if $\mathrm{K} 1$ and $\mathrm{K} 2$ are widely separated is a significant difference made by the geometry (arising because of the weaker mutual illumination of $\mathrm{K} 1$ and $\mathrm{K} 2$ ). The overall conclusion from this modeling is that for equipartition magnetic fields (where equipartition in this case 


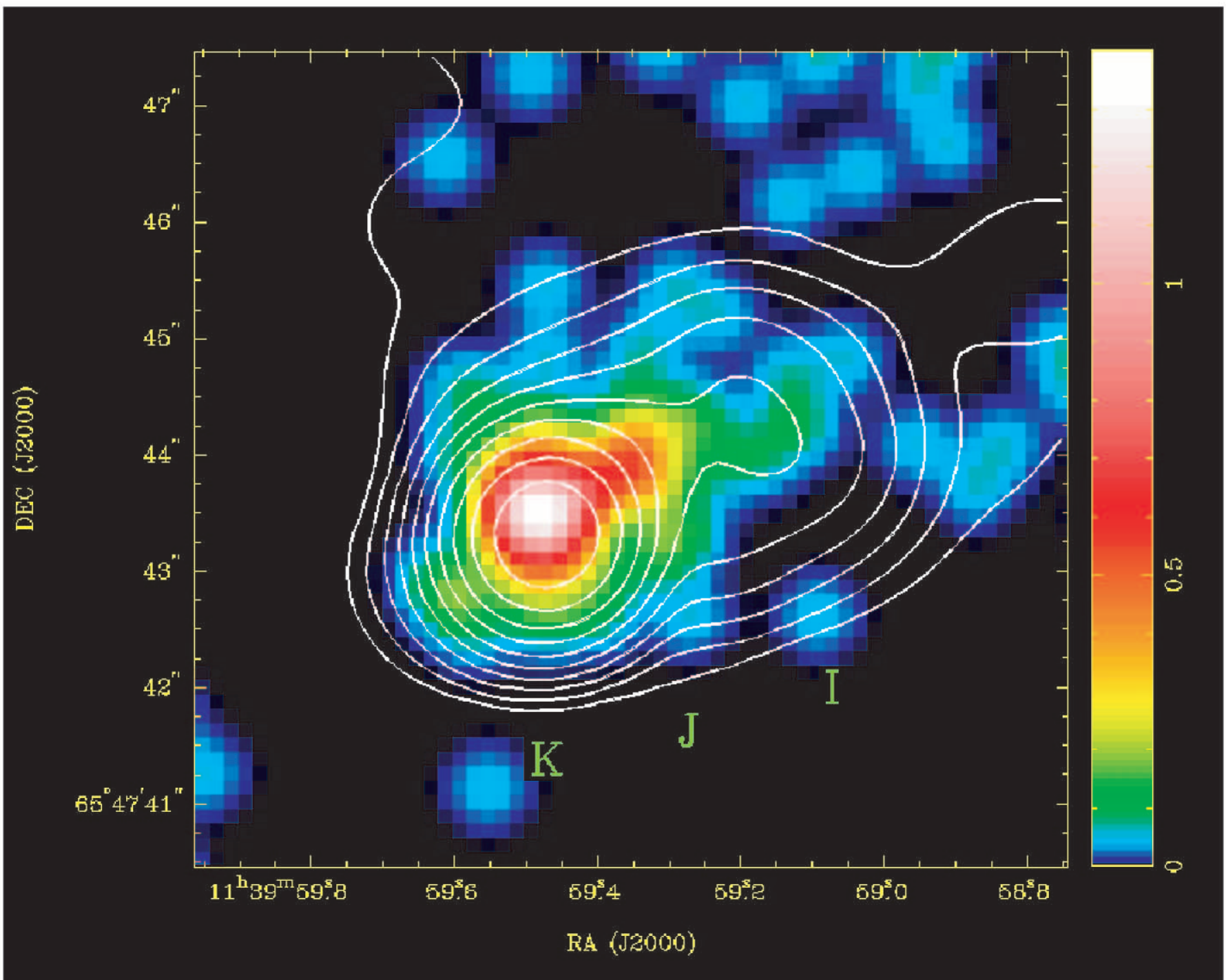

FIG. 3.-Radio and X-ray hot spot of 3C 263. The X-ray hot spot is smoothed with a 0.5 Gaussian. Overlaid are $5 \mathrm{GHz}$ radio contours at a resolution of 0.85, approximately matched to the Chandra data. These are logarithmic in steps of 2, starting at $0.5 \mathrm{mJy} \mathrm{beam}^{-1}$. The labels K, H, and I refer to features of the radio emission discussed in the text, following the notation of B94.

means that equipartition holds separately for each spectral component) the inverse Compton flux density from hot spot $\mathrm{K}$ is typically less than the observed value by a factor of 3.5. To produce the observed X-ray flux density by the inverse Compton process, the magnetic field strengths in the two components of the hot spot must be lower than the equipartition value by a factor of 1.9 (for the case where the compact component is at the center of the extended component), so that the compact component has $B=15 \mathrm{nT}$ and the extended component has $B=7 \mathrm{nT}$. The predicted $\mathrm{X}$-ray spectral index at an observed energy of $1 \mathrm{keV}$ is then 0.75 , which is consistent within the $1 \sigma$ error with the observed value, $1.0 \pm 0.3$. This conclusion is robust to changes in the model parameters; for example, even a simple homogeneous spherical model, while giving different values for the equipartition magnetic field strength, predicts a net inverse Compton flux density of $0.26 \mathrm{nJy}$ and requires a factor of $\sim 2$ decrease in field to reproduce the observed X-rays.

The comparatively strong detection of component $\mathbf{J}$ is puzzling. Its $1 \mathrm{keV}$ flux density is $\sim 0.35 \pm 0.1 \mathrm{nJy}$, assuming spectral parameters similar to those of $\mathrm{K}$. If the X-ray emission corresponds to the narrow feature seen in Figure 4, it is much too bright to be SSC at or close to equipartition. A long, thin cylinder is a very poor SSC source, and the equipartition $1 \mathrm{keV}$ flux density of this component should be $\sim 2$ pJy, including scattering of photons from K1 and K2. The magnetic field would have to be lower than equipartition by a factor of $\sim 20$ if all the X-ray flux were to be produced by component $\mathrm{J}$ by the SSC and inverse Compton processes. If there is significant relativistic motion in $\mathrm{J}$, then in its frame the emission from $\mathrm{K}$ and from the $\mathrm{CMB}$ is enhanced, and this can increase the net X-ray emissivity; however, it is hard to achieve the required increase without very large bulk Lorentz factors and small angles to the line of sight. Our model here is unrealistically simple, as it does not take account of the extended emission around $\mathrm{J}$ : however, it seems clear that this region is significantly different from hot spot $\mathrm{K}$.

The much fainter northwest compact hot spot, B in the notation of B94, is not detected in X-rays. Assuming that the source is unresolved to Chandra and using a detection 


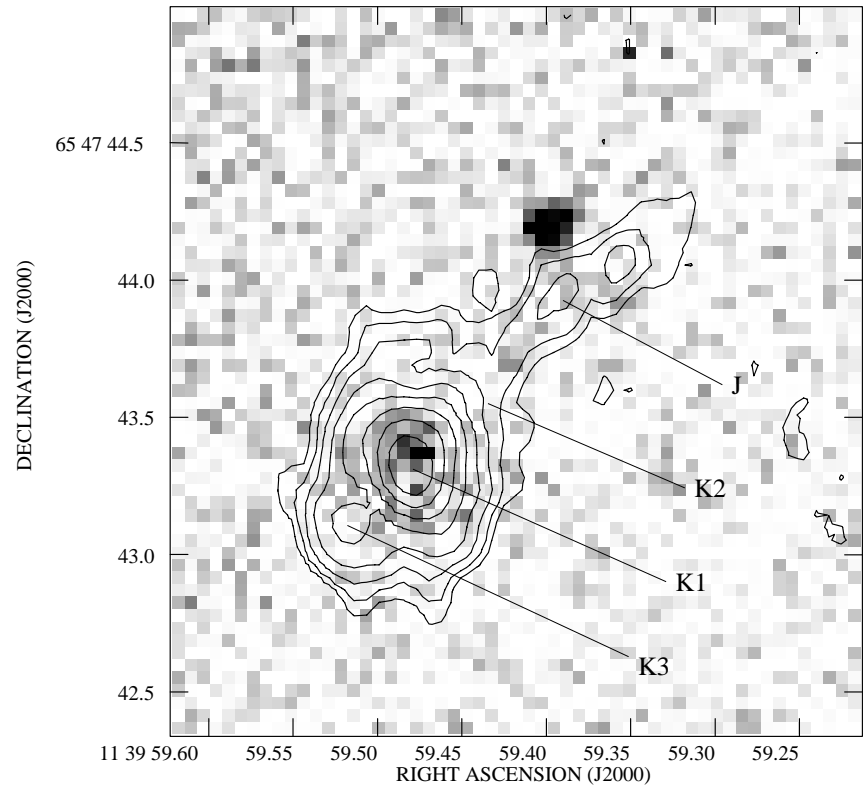

FIG. 4.-Optical hot spot of 3C 263 with high-resolution radio data. The gray scale runs between 4 and $11 H S T$ counts per PC pixel. The radio map is the $15 \mathrm{GHz}$ map at full resolution $\left(00^{\prime \prime} 14 \times 00^{\prime \prime} 10\right)$. The lowest radio contour is at $0.2 \mathrm{mJy} \mathrm{beam}^{-1}$, and the contours increase logarithmically by a factor of 2. The components $\mathrm{K} 1, \mathrm{~K} 2$, and $\mathrm{K} 3$ of hot spot $\mathrm{K}$ and the linear feature $\mathrm{J}$, discussed in the text, are labeled.

cell of six standard Chandra pixels (the $80 \%$ encircled energy criterion, as used by celldetect), we can place a $3 \sigma$ upper limit on its count rate of $1 \times 10^{-4} \mathrm{~s}^{-1}$ over the $0.4-7.0 \mathrm{keV}$ energy band, estimating the background count level from

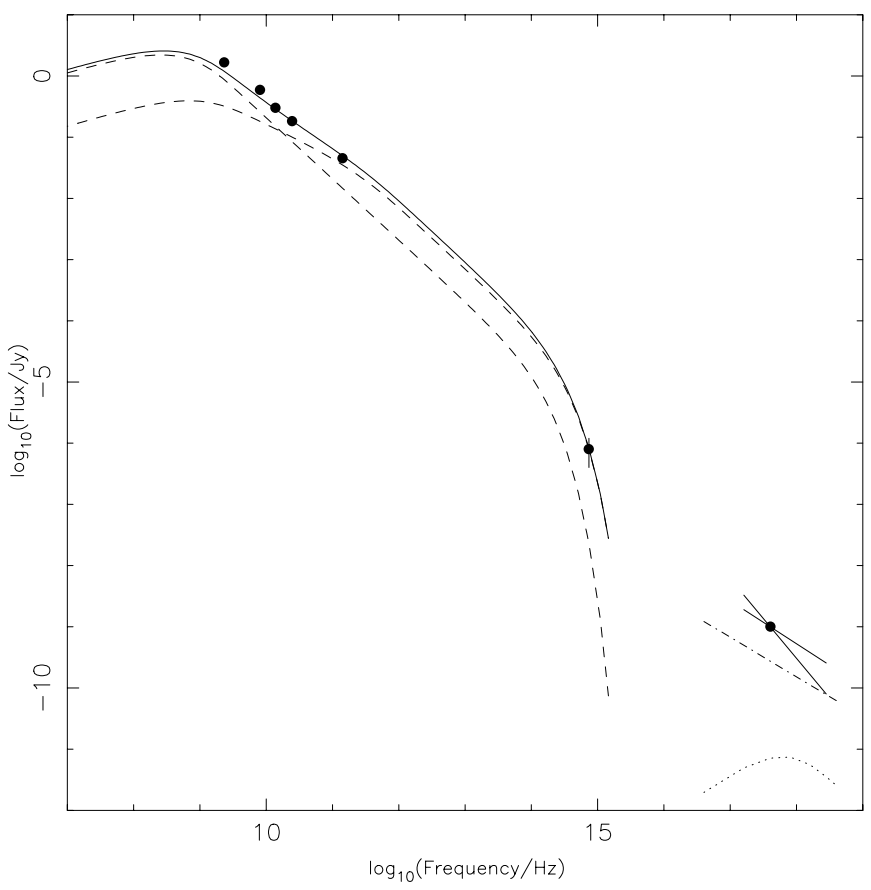

FIG. 5.-Source-frame spectrum of hot spot K of 3C 263. Radio points from Table 9, the optical HST flux density and the X-ray flux density are plotted together with the two-component synchrotron model with $\gamma_{\min }=1000$ discussed in the text (dashed lines show the two components, solid line shows the total) and the predicted total SSC emission (dot-dashed line) and $\mathrm{CMB} / \mathrm{IC}$ emission (dotted line) in this model at equipartition. the region around the hot spot, and convert this to a limit on flux density by using the spectrum of the detected hot spot. No optical counterpart to this hot spot is visible in the HST image. Its predicted inverse Compton X-ray flux density, assuming similar electron spectral parameters to those used for hot spot K, a homogenous spherical model, and an equipartition magnetic field, is $5 \mathrm{pJy}$, an order of magnitude below our upper limit. We can say only that $B$ cannot be more than a factor of $\sim 4$ below $B_{\text {eq }}$ in this hot spot.

\subsection{Jet}

There is no evidence of X-ray emission from 3C 263's jet in the data, with the possible exception of the hot spotrelated feature $\mathrm{J}$, discussed above. The jet is 11".6 long in the radio images of B94 and is essentially unresolved transversely at their highest resolution of 0.35 . Using a background region in the southeast lobe, we estimate a $3 \sigma$ upper limit on its count rate, as tabulated in Table 8 . We convert this to an upper limit on the jet X-ray flux density by using the spectrum of the detected hot spot.

The radio flux density of the jet is only $8 \mathrm{mJy}$ at $5 \mathrm{GHz}$, so that its radio to $\mathrm{X}$-ray spectral index is $\gtrsim 1.0$. If the $\mathrm{X}$-ray emission from quasar jets is boosted inverse Compton scattering from the CMB, as suggested by various authors (e.g., Tavecchio et al. 2000), then its expected X-ray flux density depends on the bulk Lorentz factor $\Gamma$ and the angle made by the jet to the line of sight $\theta$. We find the upper limit to be inconsistent only with extreme models of the jet, with $\Gamma \gtrsim 7$ and $\theta \lesssim 10^{\circ}$. The overall appearance of the source suggests that the angle to the line of sight is a good deal larger than this, in which case even larger bulk Lorentz factors are not ruled out by the X-ray data.

\subsection{Lobes}

X-ray emission is detected from the lobes of 3C 263 (Fig. 6). Although the detection of the northwest lobe emission is more obvious, because it is further from the core and there is no compact hot spot emission, we also detect excess counts from the southeast lobe.

For inverse Compton calculations we model both lobes with similar broken power-law electron distributions. We take $\gamma_{\min }$ here to be 100 rather than 1000 , to take some account of the effects of adiabatic expansion; the inverse Compton prediction is insensitive to this choice; $\gamma_{\text {break }}$ is required to correspond to a frequency near the radio region, to produce the steep spectrum of the lobes, and we model both lobes with high-energy cutoffs in the radio region, $\gamma_{\max } \sim 2 \times 10^{4}$. The predicted $1 \mathrm{keV} \mathrm{CMB} / \mathrm{IC}$ flux densities on this model (which treats the lobes as uniform) are 0.13 $\mathrm{nJy}$ for the south lobe and $0.2 \mathrm{nJy}$ for the north lobe, a factor of $\sim 4$ below the observed flux densities in both cases. The magnetic field strength in the lobes must be a factor of $\sim 2$ below the equipartition value if the lobe X-ray emission is to be produced by inverse Compton scattering of the $\mathrm{CMB}$. With this reduction in field strength, the pressures in the lobes due to electrons and magnetic field are between 1.5 and $2.0 \times 10^{-12} \mathrm{~Pa}$.

\subsection{Extended Emission}

3C 263 shows evidence for extension above the wings of the PSF out to the edge of the S3 chip (Fig. 7). There are $823_{-120}^{+64}$ net $0.5-7.0 \mathrm{keV}$ counts in the extended component, and the best-fitting model has $\beta=0.45_{-0.08}^{+0.07}, \theta_{c}=00^{\prime \prime} 9_{-0^{\prime \prime}, 9}^{+4^{\prime \prime}}$, 


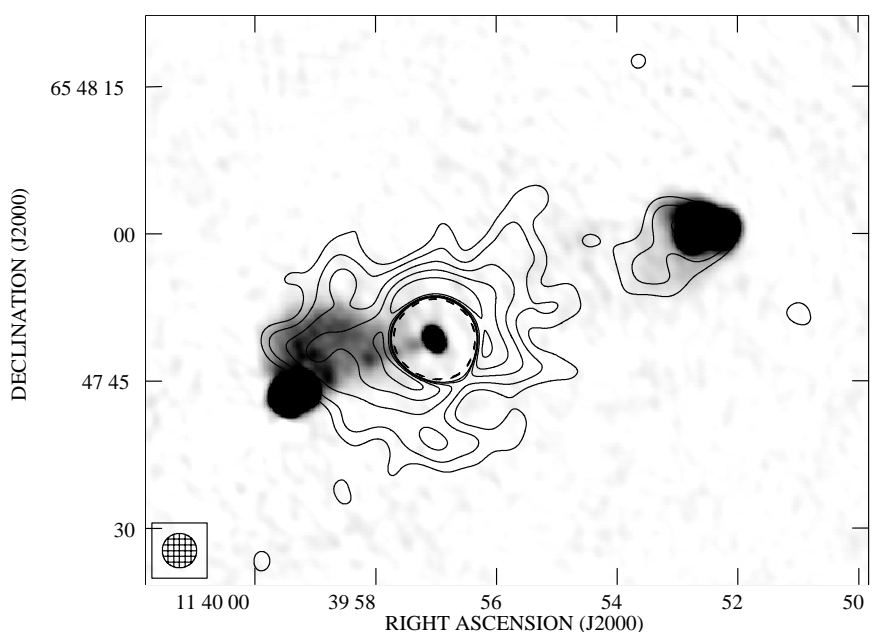

FIG. 6.-Lobes and extended emission of 3C 263. The contours show the X-ray emission smoothed with a 3".5 (FWHM) Gaussian. The hot spot regions were masked out before smoothing, and a scaled, smoothed, energy-weighted PSF model was subtracted from the image after smoothing to remove the nuclear emission. Because the X-ray core of 3C 263 is piled up, this subtraction leaves some residuals at the center of the smoothed image. The lowest contour is the $3 \sigma$ level, defined according to the prescription of Hardcastle (2000), and the contours increase logarithmically by a factor of $\sqrt{2}$. The FWHM of the convolving Gaussian is shown in the bottom left-hand corner. The gray scale shows a $1.4 \mathrm{GHz}$ VLA image with $1^{\prime \prime} .5 \times 1$ ". 1 resolution; black is $10 \mathrm{mJy}^{\text {beam }}{ }^{-1}$.

where errors are $1 \sigma$ for two interesting parameters: no lower limit is set on the core radius, so that the models are effectively power-law models outside the central regions. The background level is too high to allow us to extract a useful spectrum of the extended emission region, but assuming a temperature of a few $\mathrm{keV}$, the count rate implies a bolometric X-ray luminosity in the region, which extends out to $120^{\prime \prime}(0.9 \mathrm{Mpc})$, of $(2-3) \times 10^{44} \mathrm{ergs} \mathrm{s}^{-1}$, consistent with

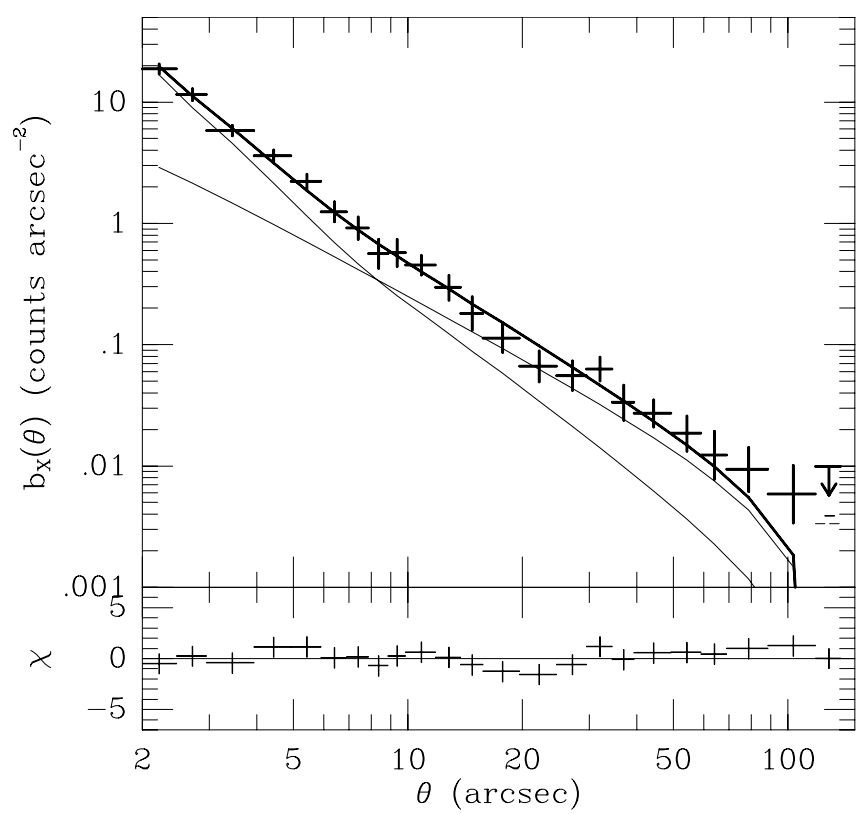

FIG. 7.-Background-subtracted radial profile of 3C 263 after hot spotand lobe-related emission has been masked out. The two light solid lines show the PSF (steeper slope) and extended model (flatter slope) fitted to the source, and the heavy line is their sum. the ROSAT-derived upper limits and with the presence of a spectroscopically confirmed cluster around 3C 263. This luminosity would correspond, on the temperatureluminosity relation for clusters (e.g., Wu, Xue, \& Fang 1999) to a temperature $k T \approx 3 \mathrm{keV}$. Using the analysis of Birkinshaw \& Worrall (1993) to convert the parameters of the $\beta$-model to physical conditions in the extended hot gas, we find that the central density on the best-fitting model would be $2 \times 10^{5} \mathrm{~m}^{-3}$ and the central pressure $1.9_{-1.7}^{+7.0} \times 10^{-10} \mathrm{~Pa}$ (errors are determined from the $\beta$-model fits and do not include temperature uncertainties). The pressure at the distance of the lobes is better constrained: at $20^{\prime \prime}$ $(150 \mathrm{kpc})$ it is $2.8_{-0.4}^{+0.3} \times 10^{-12} \mathrm{~Pa}$ for the adopted temperature. If the source is at a small angle to the line of sight, the external pressures corresponding to the lobes will be lower. These pressures are similar to the internal pressures of the lobes determined above ( $§ 4.5)$.

\section{5. $3 \mathrm{C} 330$}

\subsection{Introduction}

$3 \mathrm{C} 330$ is a narrow-line radio galaxy with $z=0.55$. Radio images of the hot spots are presented by Fernini, Burns, \& Perley (1997) and by Gilbert et al. (2002, hereafter G02). The lobes are best seen in lower resolution maps by Leahy, Muxlow, \& Stephens (1989). Optical clustering estimates (Hill \& Lilly 1991) imply a reasonably rich environment for 3C 330, but no X-ray emission was detected from the source in the off-axis ROSAT observations discussed by Hardcastle $\&$ Worrall (1999). The observations we report here are thus the first X-ray detection of 3C 330.

\subsection{Core}

3C 330's core has a relatively complex X-ray spectrum (Fig. 8). Unlike the cores of other FR II radio galaxies that we have studied with Chandra (Worrall et al. 2001b; Hardcastle et al. 2001a), it is not adequately fitted with a simple absorbed power-law model. The simplest model that gives a good fit consists of two power laws, one with Galactic absorption and one with an additional absorption column, intrinsic to the radio source, of $4 \pm 2 \times 10^{23} \mathrm{~cm}^{-2}$. This column density is comparable to that inferred from hard X-ray observations in Cygnus A (Ueno et al. 1994), although the errors are large. Such high column densities are conventionally explained as being a signature of the dense, dusty torus that is invoked in unified models to obscure the quasar nucleus and broad-line regions in narrow-line radio galaxies. In previous work (Hardcastle \& Worrall 1999, and references therein) we have argued that soft X-ray emission can arise in a component related to the radio core, through inverse Compton or synchrotron emission: this component can originate on scales larger than those of the torus and so is not heavily absorbed. In 3C 330, it seems plausible that the unabsorbed power law is this radio-related component, while the heavily absorbed component is due to the hidden quasar. 3C 330's radio core is comparatively weak (only 0.7 mJy at 5 GHz: Fernini et al. 1997), which may explain why we are able to see the heavily absorbed component in this source but not in others, where a stronger radio-related component dominates. If we remove the absorbing column, the (observer frame) $2-10 \mathrm{keV}$ flux of the absorbed component is $\sim 10^{-13} \mathrm{ergs} \mathrm{cm}^{-2} \mathrm{~s}^{-1}$ (with a large uncertainty due to the poorly constrained spectrum). This is only an order of 


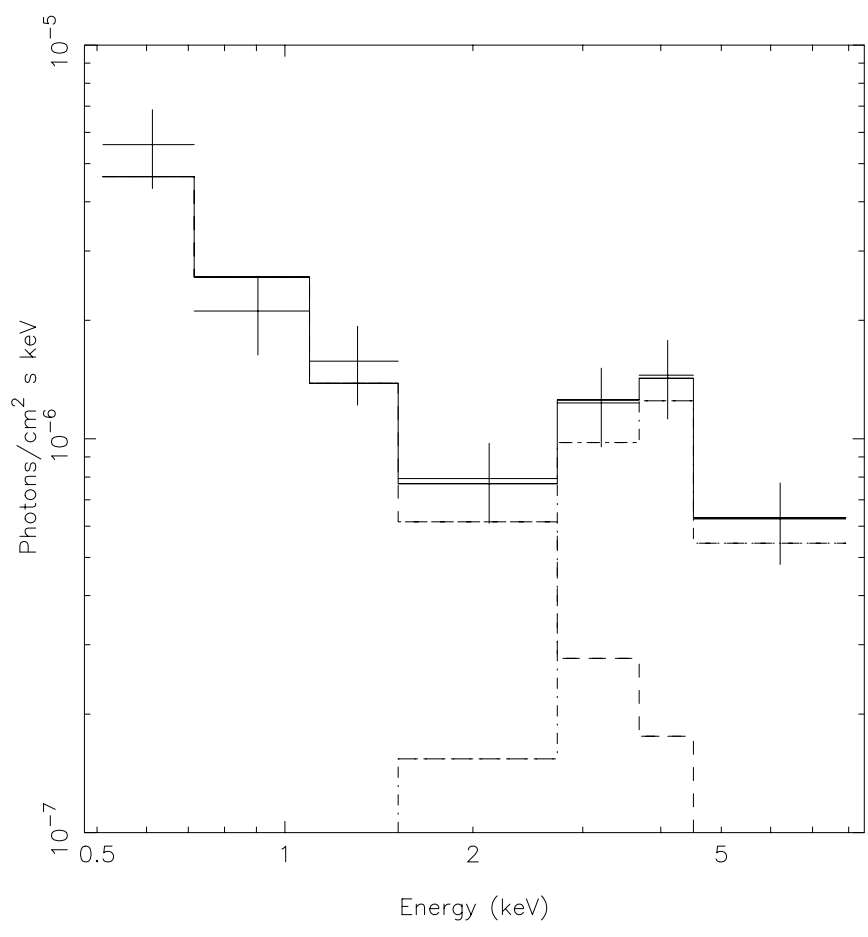

FIG. 8.-X-ray spectrum of the core of $3 \mathrm{C} 330$. Solid crosses show the $\mathrm{X}$-ray data. The lines show the model discussed in the text: the dashed line is the unabsorbed power law, the dot-dashed line is the absorbed component, and the solid line is the total model.

magnitude less than the fluxes in the same band that we determine for the two quasars in our sample, implying luminosities that are not very dissimilar, given the similar redshifts. The $1 \mathrm{keV}$ flux density for the unabsorbed component is in good agreement with the radio/soft X-ray correlation of Hardcastle \& Worrall (1999) and is consistent with the ROSAT upper limit on 3C 330's $1 \mathrm{keV}$ flux density presented there.

Because both the radio and the X-ray cores are fainter in this source than in the other sources in our sample, the radio-X-ray alignment is less certain. The X-ray centroid, using the default Chandra astrometry, is at $16^{\mathrm{h}} 09^{\mathrm{m}} 34^{\mathrm{s}} .919$, $+65^{\circ} 56^{\prime} 37^{\prime \prime} .37$, while our best radio position (from the map of G02) is $16^{\mathrm{h}} 09^{\mathrm{m}} 34 \mathrm{~s} .896,+65^{\circ} 56^{\prime} 37^{\prime \prime} \cdot 69$, in close agreement with the position found by Fernini et al. (1997). This implies a radio-X-ray offset of 0 " 35 , and we correct the X-ray data accordingly.

\subsection{Hot Spots}

Figure 9 shows that the northeast radio-bright hot spot of 3C 330 is clearly detected in the X-ray. There is also a weak but significant detection of the southwest hot spot. The $\mathrm{X}$-ray peak of the brighter hot spot agrees well with the radio flux peak, and it may be marginally extended along the same axis as the bright radio-emitting region.

There are insufficient net counts even in the brighter hot spot to extract a spectrum. We convert the count rates to 1 $\mathrm{keV}$ flux densities on the basis of a power-law spectrum with $\alpha_{\mathrm{X}}=0.5$ and Galactic absorption. The conversion factor is not sensitive to the precise choice of spectral index.

Neither hot spot is detected in the HST observation. We set a $3 \sigma$ upper limit on each hot spot's optical flux density of $0.5 \mu \mathrm{Jy}$ at $5.5 \times 10^{14} \mathrm{~Hz}$.
The spectrum of the northeast hot spot of $3 \mathrm{C} 330$ is plotted in Figure 10. The structure of this hot spot is quite well resolved by the high-resolution image of G02; it can be modeled adequately as a cylinder with length 2 ". 3 and radius 0.64 with a linear intensity gradient along its length (which we model as a linear increase in electron spectral normalization).

The spectrum of the hot spot at radio frequencies is steep ( $\alpha \approx 1.0$ ), and, since we only have one high-resolution map and cannot resolve any spectral structure of the hot spot, we simply model it as a single broken power-law model with the break between spectral indices of 0.5 and 1.0 occurring close to $1.4 \mathrm{GHz}$. For equipartition, $\gamma_{\text {break }}=3000$. We set $\gamma_{\min }=1000$. The best fit to the radio observations, including the high-frequency BIMA data, is well-fitted with a model with $\gamma_{\max }=1.4 \times 10^{4}$; the spectral index between 15 and $85 \mathrm{GHz}$ is steeper than 1.0 , which requires the cutoff to be at low energies. In this model, the equipartition field strength is $9.5 \mathrm{nT}$, and the predicted SSC flux density is 0.49 $\mathrm{nJy}$. If the hot spot is not in the plane of the sky, projection means that the actual long axis of the hot spot is longer than we have assumed, and the predicted SSC emissivity is reduced, by about $10 \%$ for an angle to the line of sight of $45^{\circ}$. Given these uncertainties, we can say that the observed SSC emission in the northeast hot spot, with a $1 \mathrm{keV}$ flux density of $0.34 \pm 0.07 \mathrm{nJy}$ on simple spectral assumptions, implies a magnetic field equal to or slightly higher than the equipartition value.

The compact component of the southeast hot spot is resolved from the others only at $8 \mathrm{GHz}$, so we cannot measure its spectrum; we assume the same electron spectral values as for the northeast hot spot, with a spherical model. The predicted $1 \mathrm{keV}$ SSC flux density from this component at equipartition $(B=18 \mathrm{nT})$ is then between 0.03 and $0.04 \mathrm{nJy}$, depending on the value of $\gamma_{\max }$. This is consistent with the observed flux density of $0.09 \pm 0.04 \mathrm{nJy}$, given the large uncertainties, but the observations are better fitted with a magnetic field strength a factor of $\sim 1.5$ below equipartition.

\subsection{Lobes}

3C 330's lobes are both clearly detected in the X-ray (Fig. 11). We defined rectangular extraction regions that avoid the core (starting at about $10^{\prime \prime}$ away from it) and the hot spots, using parallel adjacent rectangles on either side to give a local background subtraction. The lobes each contain about 20 net counts in the $0.4-7.0 \mathrm{keV}$ range, so it is not possible to extract useful spectra. As with the hot spots, we convert the count rate to $1 \mathrm{keV}$ flux density on the basis of a power law with $\alpha_{\mathrm{X}}=0.5$ and Galactic absorption.

We model the lobes with the same spectral assumptions as for 3C 263 (§ 4.5). In this case the predicted $1 \mathrm{keV}$ flux densities from $\mathrm{CMB} / \mathrm{IC}$ at equipartition are 0.17 and 0.18 $n J y$. Given the large errors on the measured lobe fluxes, we cannot rule out equipartition in this case; taking the measured fluxes at face value, they imply magnetic field strengths a factor of $\sim 1.4$ below equipartition. These would imply internal lobe pressures from electrons and magnetic field of around $1.5 \times 10^{-12} \mathrm{~Pa}$.

\subsection{Extended Emission}

3C 330 has weak extended emission (Fig. 12), with the fits showing evidence for only $186_{-37}^{+44}$ extended counts out to $70^{\prime \prime}(0.5 \mathrm{Mpc})$. The best-fitting $\beta$ model has $\beta=0.65_{-0.18}^{+1.3}$, 

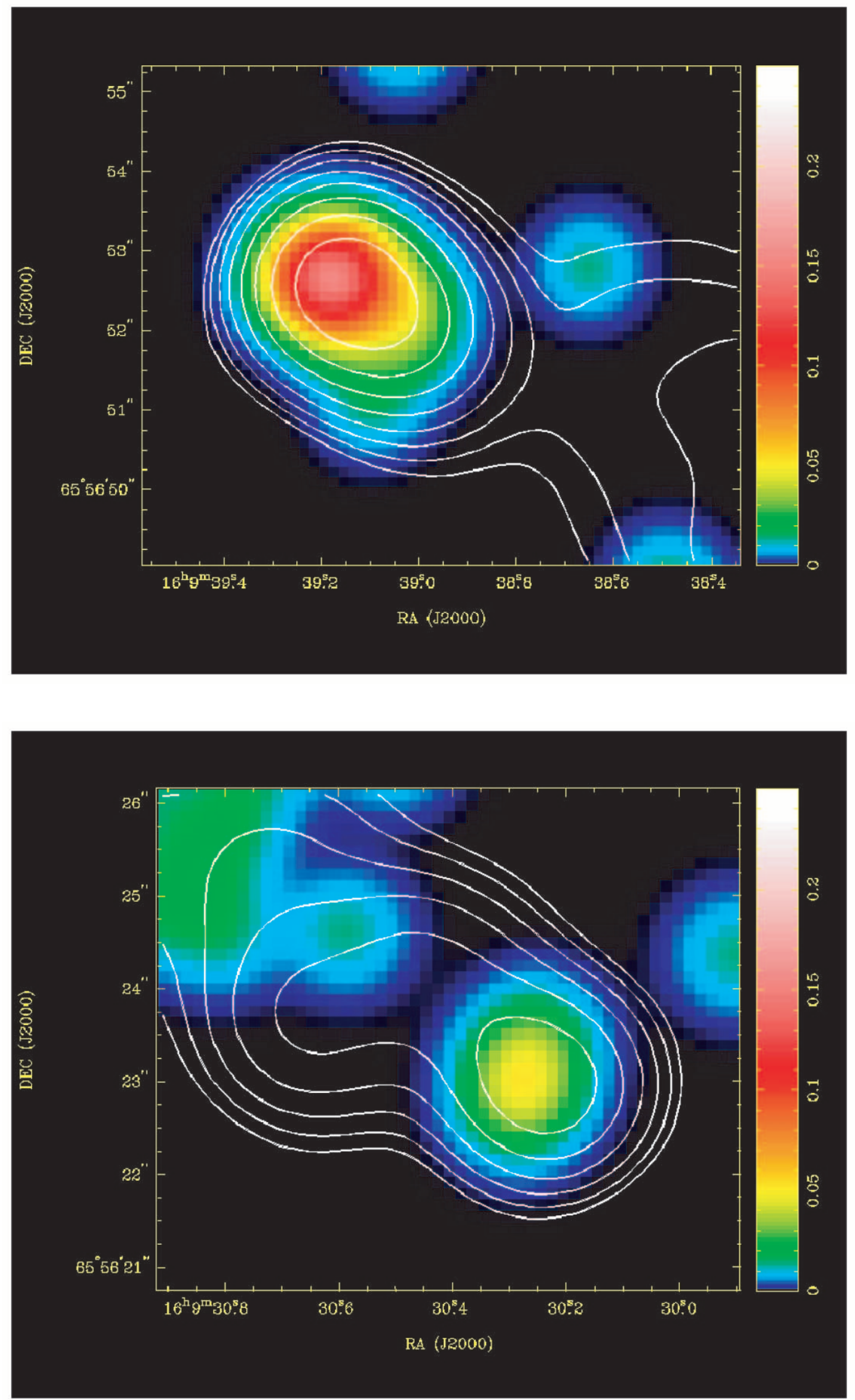

FIG. 9.-X-ray hot spots of 3C 330 smoothed with a $1^{\prime \prime}$ Gaussian. Contours in both cases are from an $8.4 \mathrm{GHz}$ radio map with a resolution of 1 "'2, approximately matched to the X-ray resolution. The contours increase logarithmically by a factor of 2 and the lowest contour is at $1 \mathrm{mJy}^{\text {beam }}{ }^{-1}$. Left: northeast hot spot; right: southwest hot spot. 


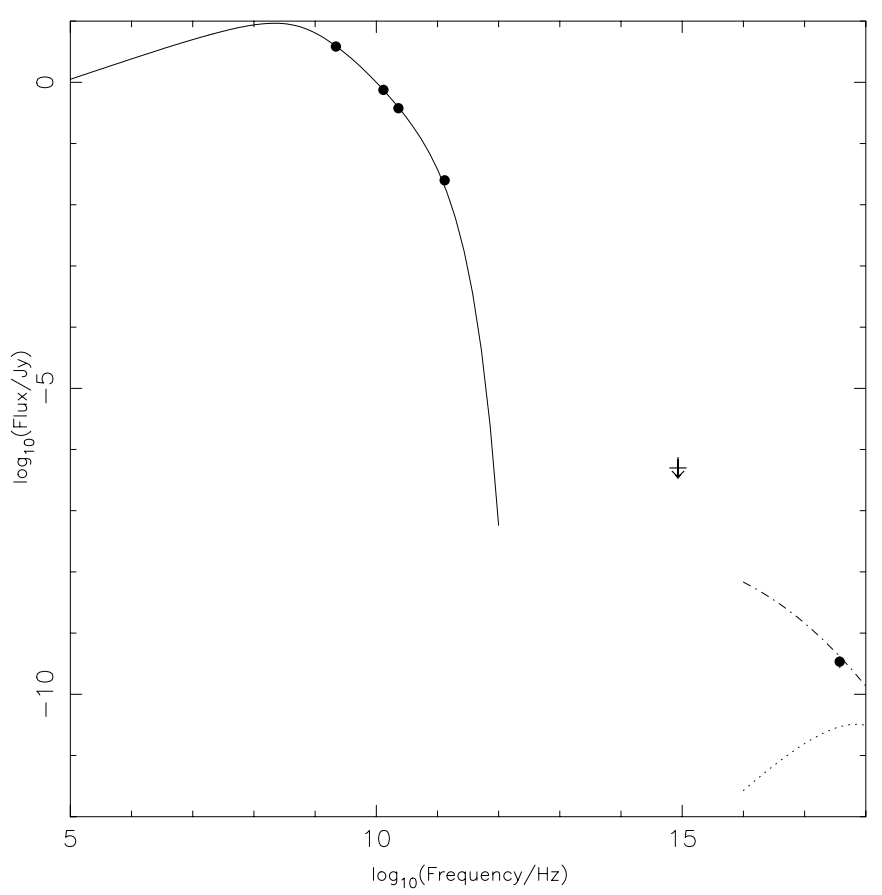

FIG. 10.- Source-frame spectrum of the north hot spot of 3C 330. Radio points from Table 9, the optical $H S T$ flux density limit and the X-ray flux density are plotted together with the best-fitting synchrotron model with $\gamma_{\min }=1000$ (solid line) and the predicted SSC (dot-dashed line) and $\mathrm{CMB} / \mathrm{IC}$ (dotted line) emission from the hot spot at equipartition. The high-energy cutoff is set to $\gamma_{\max }=1.4 \times 10^{4}$ in this figure.

$\theta_{c}=5^{\prime \prime} 9_{-3.7}^{+15}$ (errors are $1 \sigma$ for two interesting parameters). The luminosity of this extended component is then around $3 \times 10^{43} \mathrm{ergs} \mathrm{s}^{-1}$, corresponding to a temperature around $1.5 \mathrm{keV}$. In the best-fitting model, the central density is $\sim 2 \times 10^{4} \mathrm{~m}^{-3}$ and the central pressure $8.4_{-3.8}^{+9.1} \times 10^{-12} \mathrm{~Pa}$.

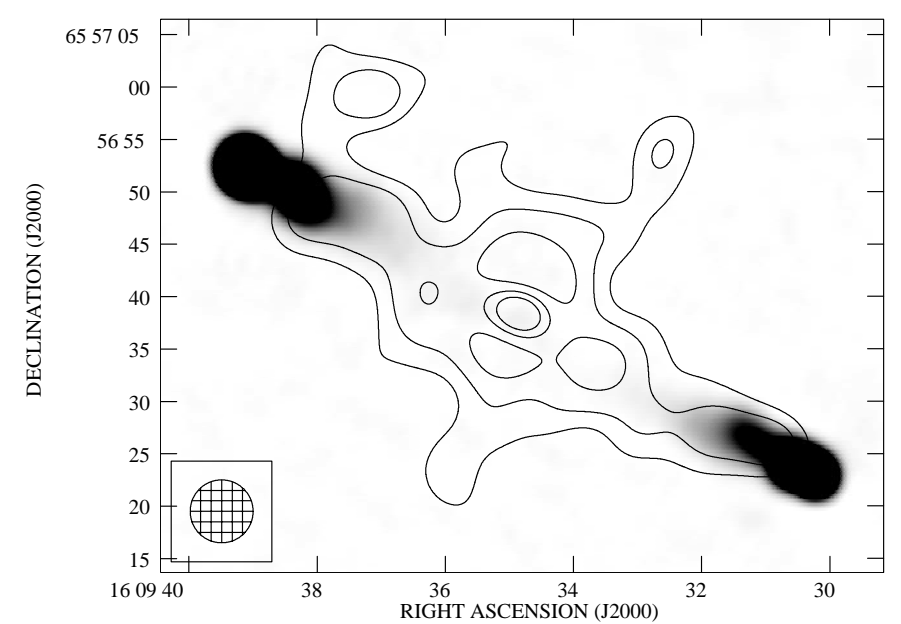

FIG. 11.-Lobes and extended emission of $3 \mathrm{C} 330$. The contours show the X-ray emission smoothed with a 6" (FWHM) Gaussian. The hot spot regions were masked out before smoothing and a scaled, smoothed, energyweighted PSF model was subtracted from the image after smoothing to remove the nuclear emission. The lowest contour is the $3 \sigma$ level, defined according to the prescription of Hardcastle (2000), and the contours increase logarithmically by a factor $\sqrt{2}$. The FHWM of the Gaussian used to convolve the X-ray emission is shown in the bottom left-hand corner. The object to the northwest of the northeast hot spot seems to be a faint background X-ray source. The gray scale shows the $8.4 \mathrm{GHz}$ VLA image with $3^{\prime \prime}$ resolution of G02; black is $10 \mathrm{mJy} \mathrm{beam}^{-1}$.

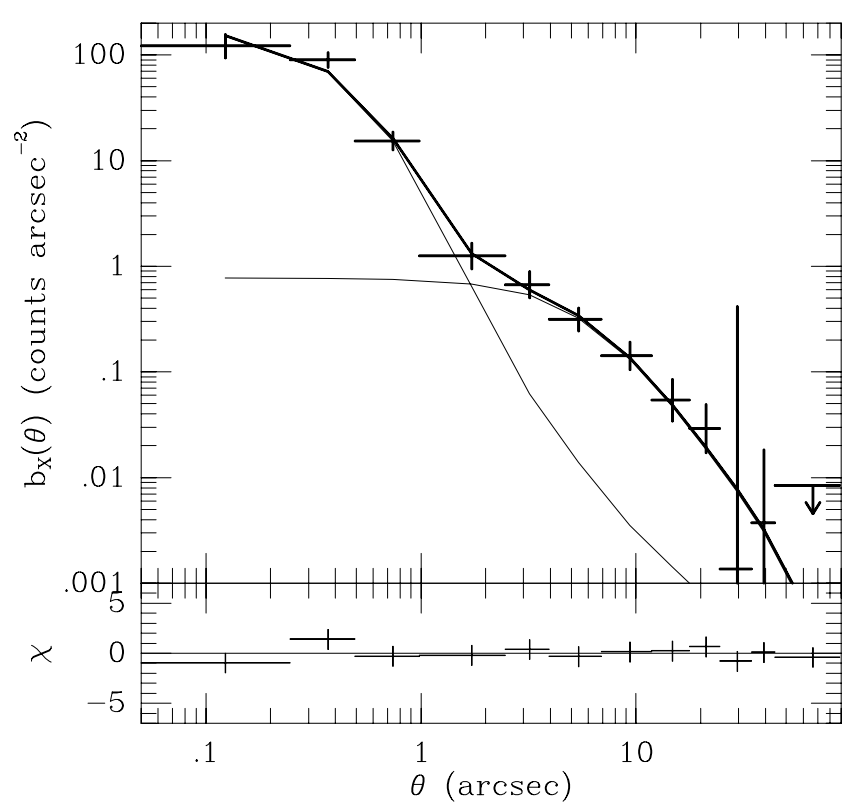

FIG. 12.-Background-subtracted radial profile of 3C 330 after hot spotand lobe-related emission has been masked out. The two light solid lines show the PSF (smaller scale) and extended model (larger scale) fitted to the source, and the heavy line is their sum.

The pressure at distances corresponding to the lobes, assuming little projection of this source, varies from $2.2_{-0.3}^{+0.4} \times 10^{-12} \mathrm{~Pa}$ (at $10^{\prime \prime}, 70 \mathrm{kpc}$ ) to $3.3_{-2.0}^{+1.0} \times 10^{-13} \mathrm{~Pa}$ (at $30^{\prime \prime}, 210 \mathrm{kpc}$ ). These are similar to the estimate of the internal lobe pressure above: the lobes would be in pressure balance at a radius of around $10^{\prime \prime}$ but could be overpressured with respect to the external medium at larger distances.

\section{3 C 351}

\subsection{Introduction}

3C 351 is a well-studied $z=0.37$ quasar. Radio images have been presented by Leahy \& Perley (1991), B94, and G02; their most striking feature is the bright double hot spot pair to the north and the displaced nature of the north lobe. The hot spots are also detected in the optical (Röser 1989; Lähteenmäki \& Valtaoja 1999). The X-ray emission from the bright nucleus has been extensively studied (Fiore et al. 1993; Mathur et al. 1994; Nicastro et al. 1999), and it has been argued that the unusual X-ray spectrum seen in ROSAT PSPC data is due to an ionized absorber, often seen in Seyfert 1 galaxies but a rare feature in quasars. No extended X-ray emission was detected with ROSAT (Hardcastle \& Worrall 1999), but the hot spots were seen in a short Chandra observation (Brunetti et al. 2001).

\subsection{Core}

The X-ray centroid, using the default Chandra astrometry, is at $17^{\mathrm{h}} 04^{\mathrm{m}} 41^{\mathrm{s}} \cdot 326,+60^{\circ} 44^{\prime} 30^{\prime \prime}$ '22. 3C $351^{\prime}$ 's radio core contains two compact components of similar $8 \mathrm{GHz}$ flux density separated by 0.35 . B94 and G02 both argue that the southern component is the true core, while the northern component is a jet knot. The radio position of the southern component, using the $8 \mathrm{GHz}$ radio map of $\mathrm{G} 02$, is $17^{\mathrm{h}} 04^{\mathrm{m}} 41^{\mathrm{s}} .364,+60^{\circ} 44^{\prime} 30^{\prime \prime} 46$. This is in good agreement with the position given by $\mathrm{B} 94$ and implies a radio-X-ray 
misalignment of 0 ". 37 . We shift the X-ray data to align the $\mathrm{X}$-ray core with the southern radio component. However, it is possible that some of the X-ray emission observed from the core region comes from the northern radio component, since the two components would not be well resolved by Chandra. We see no evidence for extended emission from the X-ray core, and no difference between the core centroids in different X-ray energy ranges, but the possibility means that the alignment of the radio and X-ray frames is less secure than it would otherwise be.

We obtain many counts in the X-ray core, and so detailed spectroscopic analysis is possible. Unsurprisingly, in view of previous work, a single power-law model with Galactic absorption is an unacceptable fit to our data $\left(\chi^{2} / n=638 / 215\right.$, where $n$ denotes degrees of freedom); we see strong residuals at $0.6-0.8 \mathrm{keV}$. A two-power-law model, with one of the power-law components having additional intrinsic absorption (as used above for 3C 330) is a better fit, with $\chi^{2} / n=304 / 212$ and an intrinsic cold absorbing column of $1.1 \pm 0.1 \times 10^{22} \mathrm{~cm}^{-2}$; but the best-fitting spectral index of the unabsorbed component in this model is well constrained and steep, $\alpha=2.5 \pm 0.3$. The absorbed power law has $\alpha=0.46 \pm 0.05$. This model is plotted in Figure 13. If the absorbed component is associated with the active nucleus and the unabsorbed component with the radio emission, then the absorbing column, which represents only 2 mag of visual extinction, is small enough to be compatible with the predictions for a quasar within unified models, while the $1 \mathrm{keV}$ flux density of the unabsorbed component would be $14 \mathrm{nJy}$, which is consistent with the radioX-ray relation of Hardcastle \& Worrall (1999).
We fit two alternative models, consisting of a single power law with Galactic absorption plus either "windowed" absorption (the zwndabs model in XSPEC), which roughly mimics a warm absorber, or the XSPEC ionized absorber model (absori). The zwndabs model gives a less good fit than the power-law models, with $\chi^{2} / n=344 / 213$; it has a window energy (source-frame) of $0.78 \pm 0.01 \mathrm{keV}$ and an absorbing column of $3.7 \pm 0.2 \times 10^{21} \mathrm{~cm}^{-2}$, and the bestfitting power-law index is very flat $(\alpha=0.10 \pm 0.02)$. The ionized absorber model gives a slightly better fit, $\chi^{2}=322 / 213$, with the ionizing continuum power-law index fixed to $\alpha_{i}=0.5$, the absorber temperature set to $3 \times 10^{4} \mathrm{~K}$ and the iron abundance set to unity. In this model, shown in Figure 13, the power-law index $\alpha=0.42 \pm 0.04$, the absorbing $N_{\mathrm{H}}=1.5 \pm 0.1 \times 10^{22}$ $\mathrm{cm}^{-2}$, and the ionization parameter $\xi=34 \pm 5 \mathrm{ergs} \mathrm{cm}^{-1}$. This absorbing column is consistent with the value obtained (under a slightly different model) by Nicastro et al. (1999).

None of these models is a particularly good fit to the data, with particularly strong scatter about the models to be seen below $0.6 \mathrm{keV}$ (Fig. 13). In Table 8 we tabulate the results for the two-power-law models, as they remain formally the best fits. Two weak linelike features are seen in the spectrum, at observed energies of about 3.2 and $4.7 \mathrm{keV}$ (Fig. 13). When these are fitted with Gaussian models, the first feature's width is not well constrained, and it is best fitted with very broad lines. This reduces our confidence in its physical reality as an emission feature. The second feature can be modeled as a Gaussian with rest-frame energy $6.40 \pm 0.07 \mathrm{keV}$ and $\sigma=70 \pm 70 \mathrm{eV}$, with equivalent width $70 \mathrm{eV}$. The improvement to the fit of adding this feature is
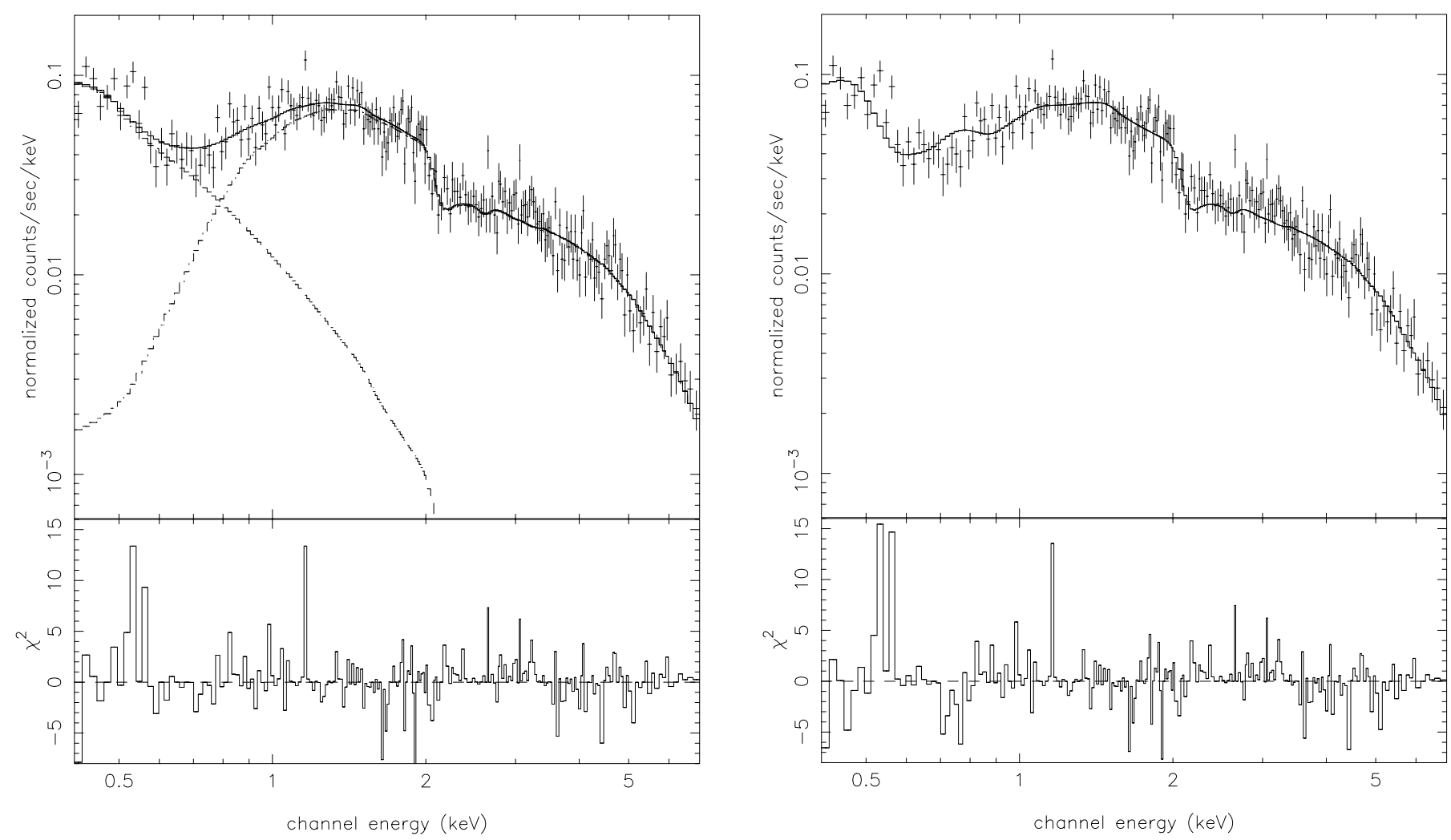

FIG. 13.-X-ray spectrum of 3C 351 nucleus. Left: The spectrum with the best-fitting two-power-law model. Right: The spectrum with the best-fitting ionized-absorber model. The lower panels show the contributions to $\chi^{2}$, plotted with the sign of the residual. 
very limited, but given its energy it may be a marginal detection of the iron $\mathrm{K} \alpha$ line.

\subsection{Hot Spots}

As previously reported by Brunetti et al. (2001), both of the northern hot spots of 3C 351 are detected in the X-ray (Fig. 14); the compact "primary" hot spot J and the diffuse "secondary" hot spot L (using the notation of B94). In our observations 5 times more counts are obtained than in the data set used by Brunetti et al., giving us well-constrained hot spot spectra (Table 8). We obtain $1 \mathrm{keV}$ flux densities and spectral indices that agree with the values determined by Brunetti et al. within the joint $1 \sigma$ uncertainties.

When these hot spots are compared with a radio map (Fig. 14) there is a clear offset of about $1^{\prime \prime}$ between the X-ray and radio peaks in the secondary hot spot $\mathrm{L}$, in the sense that the distance between the peaks of $\mathbf{J}$ and $\mathrm{L}$ is smaller in the X-ray than in the radio. $\mathrm{L}$ appears to be resolved by
Chandra and to be comparable in size to its radio counterpart $\left(\sim 2^{\prime \prime}\right)$. There is no clear color gradient in the X-ray images of $\mathrm{L}$ and no obvious radio spectral index gradient at high frequency. There is an extension of $\mathbf{J}$ to the north with no radio counterpart, but it is otherwise not resolved by Chandra.

Optical counterparts to both northern hot spots in 3C 351 were discovered by Röser (1989) and their relationship to the radio emission confirmed by Lähteenmäki \& Valtaoja (1999). Further optical observations were made by Brunetti et al. (2001), who also discuss the HST observations that we use in this paper. When the HST observations are compared with high-resolution radio maps, it appears that the optical counterpart of hot spot L may be offset from the radio peak in the same sense as is seen in the X-ray (Figs. 14 and 15). The two optical hot spots unfortunately lie on different chips of the HST WFPC2, and there are no reference sources in the PC chip to tie the radio and optical frames together. Significant offsets are present using the default

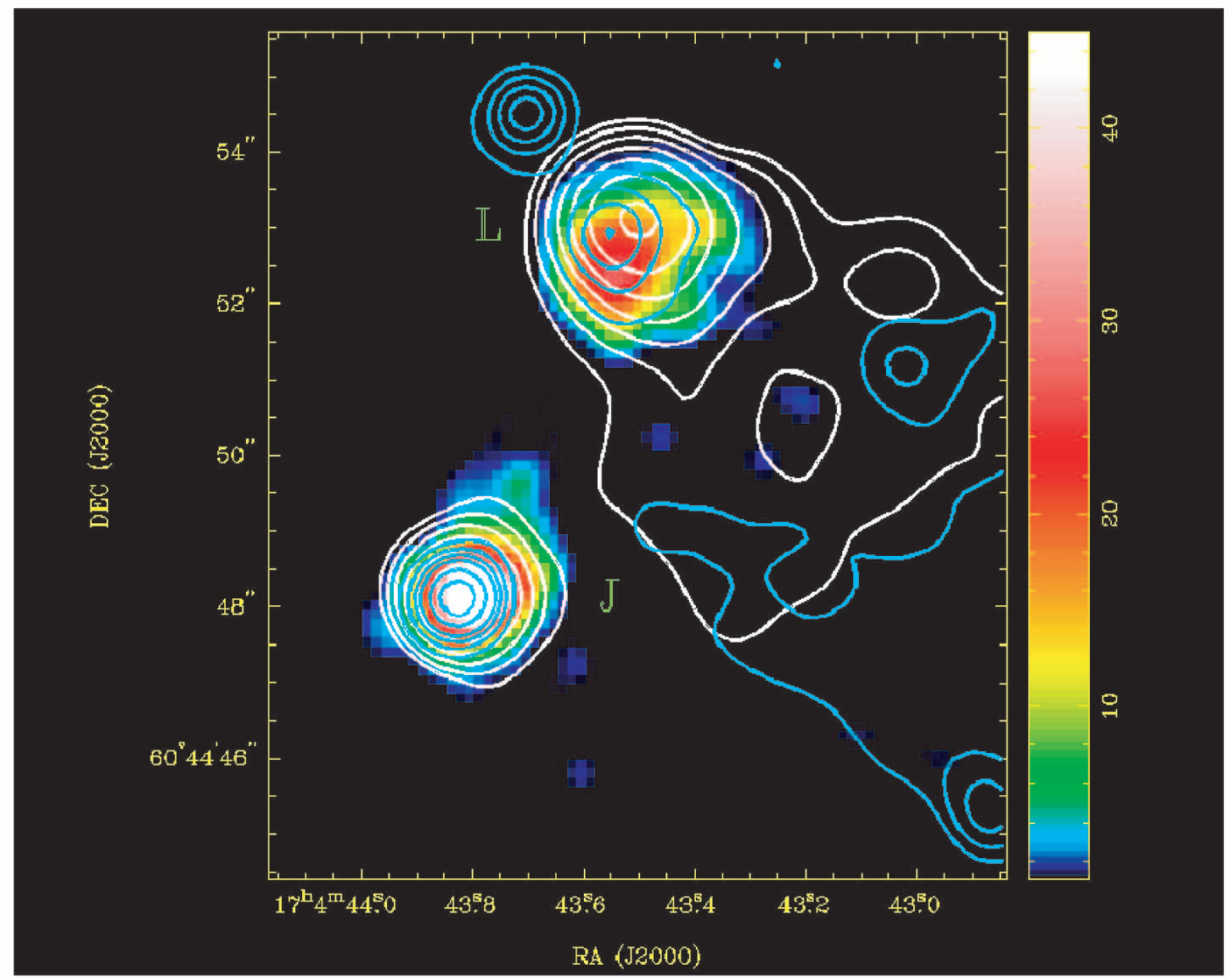

FIG. 14.-3C 351 X-ray hot spots. The Chandra data are smoothed with an 0".5 Gaussian. The white contours are 8.4 GHz radio contours with a resolution of 0 ." 85 , approximately matched to the X-ray resolution. The contours increase logarithmically by a factor of 2 , and the lowest contour is at $0.25 \mathrm{mJy}$ beam ${ }^{-1}$. The light blue contours are the HST R-band data at the same resolution, and also increase logarithmically by a factor of 2 (arbitrary units). The structure in the bottom right of the image is due to noise on the HST PC chip. 


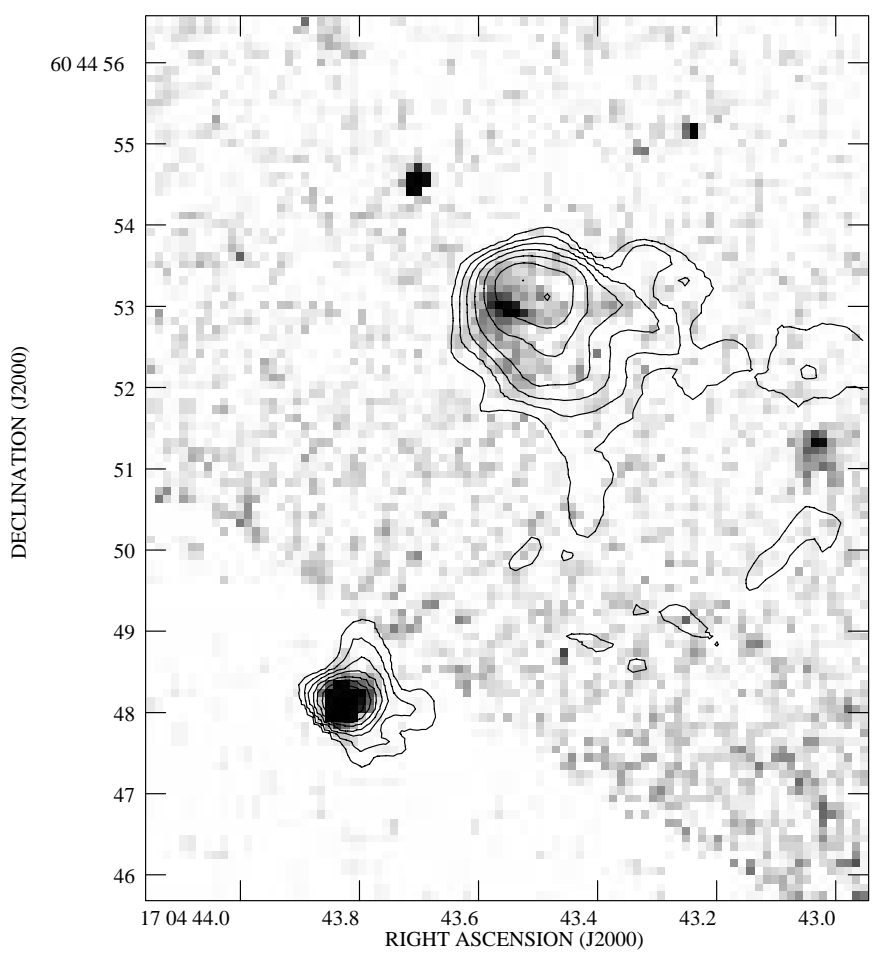

FIG. 15.-3C 351 optical hot spots at the full HST resolution. The HST data are shifted so that the positions of hot spot $\mathbf{J}$ align in the optical and radio. The contours are from an $8.4 \mathrm{GHz}$ radio map at $0.34 \times 0$ ". 23 resolution. The WFC chip lies in the bottom left of this image, the PC chip in the top right.

HST astrometry, as expected. The evidence for an offset relies on the accuracy of the wmosaic task in the STSDAS package in IRAF, used to produce the mosaiced image shown in these figures. However, the size of the offset $(0 . .5)$ seems too large to be accounted for by uncertainties in the WFPC2 chip geometry; other sources that span chip boundaries in this field (notably some faint galaxies near the quasar nucleus) do so without showing any evidence for such offsets. We conclude that the observed offset may well be real. In this case, the optical peak of $\mathrm{L}$ lies between the radio and $\mathrm{X}$-ray peaks.

We agree, within the errors, with the HST $R$-band flux density measured by Brunetti et al. (2001) for hot spot J. However, we obtain a substantially lower flux density for hot spot L, although the errors are comparatively large because of uncertainties in background subtraction. We adopt flux densities of $2.4 \pm 0.1 \mu \mathrm{Jy}$ and $1.9 \pm 0.2 \mu \mathrm{Jy}$ for $\mathbf{J}$ and $\mathrm{L}$, respectively.

The compact hot spot of $3 \mathrm{C} 351(\mathrm{~J})$ is not well resolved at any radio frequency available to us, and it is compact even in the HST images. The size derived from fitting a homogeneous sphere model depends on the frequency and resolution of the data used, suggesting that there may be unresolved spatial or spectral structure. The value we adopt $(r=0$ ". 16) is based on fitting a homogeneous sphere model to the $8.4 \mathrm{GHz}$ data of $\mathrm{G} 02$. This is larger than the value used by Brunetti et al. (2001), who took just half the FWHM of the Gaussian fit of B94. Spectrally, the hot spot seems to have a steeper spectrum between 1.4 and $8 \mathrm{GHz}$ than between 8 and $15 \mathrm{GHz}$, but this may be due to contamination from more extended emission in the low-resolution 1.4 $\mathrm{GHz}$ map; the maps of B94 and G02 both show structure around this hot spot. The high-frequency $(15-85 \mathrm{GHz})$ spectral index of the hot spot is close to 1.0. If we assume that some of the $1.4 \mathrm{GHz}$ flux density is not from the compact region, then the $8.4,15$, and $85 \mathrm{GHz}$ and optical data points can be fitted, though not particularly well, with a version of our standard spectral model, in which the synchrotron spectral index steepens from 0.5 to 1.0 in the radio and then retains this value out to beyond the $B$ band. Since the optical to X-ray spectral index is close to 1.0 , the $1 \mathrm{keV} X$-ray data point alone can then be fitted as an extension of the synchrotron spectrum, with $\gamma_{\max }>6 \times 10^{7}$ at equipartition (this conclusion differs from that of Brunetti et al., who find a somewhat lower maximum contribution from synchrotron emission, because of the steeper injection index they use). However, the flat X-ray spectrum of the hot spot $\left(\alpha_{\mathrm{X}}=0.5 \pm 0.1\right)$ is inconsistent with a synchrotron model. Like Brunetti et al., we prefer a synchrotron model in which the break is at higher frequencies and the synchrotron emission is cutting off in the optical $\left(\gamma_{\text {break }}=5800\right.$, $\gamma_{\max }=1.3 \times 10^{6}$ at equipartition). This improves the fit to the radio and optical data. However, in this model, plotted in Figure 16, the predicted inverse Compton flux density at equipartition $\left(B_{\mathrm{eq}}=21 \mathrm{nT}\right)$ is $55 \mathrm{pJy}$, almost 2 orders of magnitude below the observed value. The magnetic field must be reduced by a factor of $\sim 12$, to $1.7 \mathrm{nT}$, to produce the observed emission from the SSC process. In this case $\gamma_{\max }=4.8 \times 10^{6}, \gamma_{\text {break }}=2.0 \times 10^{4}$, and the predicted $\mathrm{X}$-ray spectral index at $1 \mathrm{keV}$ is 0.6 , close to the observed value.

Hot spot $\mathrm{L}$ is well resolved in the radio and HST images. A direct measurement on the radio map shown in Figure 14 gives dimensions for the bright region of about 2 ". $2 \times 1$ ". 6 . No simple geometrical model is a good fit to the structure of this hot spot, with its off-center brightness peak and filamentary extensions to the southwest. We begin by treating the brightest part of the hot spot as a homogeneous sphere with $r=0$ ". 8 ; this radius agrees both with the high-resolution measurements and with fits to the low-resolution 1.4 and 15 $\mathrm{GHz}$ maps, but is again somewhat larger than that used by Brunetti et al. The radio and optical data can then be fit with a spectral model very similar to that used for hot spot $\mathrm{J}$; the $1.4 \mathrm{GHz}$ data point again lies above the extrapolation of the spectrum inferred from the high-frequency radio data, but neglecting this, we find very similar break and cutoff values, $\gamma_{\text {break }}=8400, \gamma_{\max }=1.3 \times 10^{6}$, at equipartition $\left(B_{\text {eq }}=7.1 \mathrm{nT}\right)$. This model is plotted in Figure 16. If the $\mathrm{X}$-ray emission is SSC, the predicted inverse Compton flux density in this model is $80 \mathrm{pJy}$, and the magnetic field strength would have to be reduced by a factor of $\sim 9$, to 0.8 $\mathrm{nT}$, to produce all the observed emission by the inverse Compton process. The predicted $1 \mathrm{keV}$ X-ray spectral index in this model is also 0.6 , which is somewhat flatter than the observed value of $0.85 \pm 0.1$.

The inverse Compton model cannot explain the observed offsets between the X-ray and radio centroids of hot spot L. To explain this offset while retaining a simple model of the electron distribution we would need an external illuminating source, but no such source is apparent; in particular, hot spot $\mathrm{J}$ is much too far away to produce a significant effect (the predicted flux density from IC scattering of J's photons by $\mathrm{L}$ is only $2 \mathrm{pJy}$ at equipartition, or $2 \%$ of the predicted $\mathrm{SSC}$ flux density). Otherwise, if the X-rays are to be SSC emission, there must be significant electron spectral structure in the hot spot that we have failed to take into account 

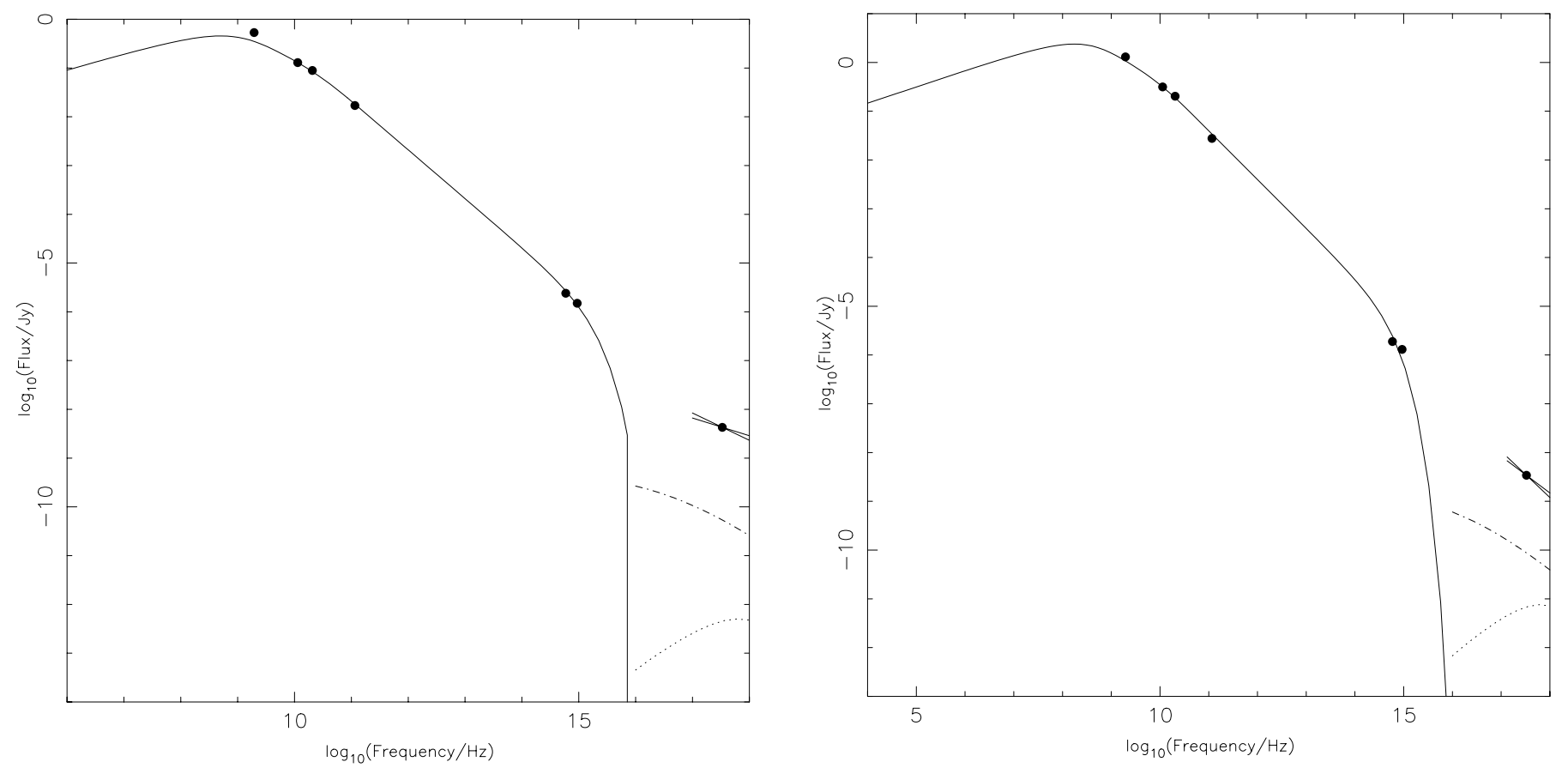

FIG. 16.- Source-frame spectra of the north hot spots of 3C 351: left is hot spot J, and right is hot spot L. Radio points from Table 9, the R-band HST flux densities determined by us, the ground-based $B$-band flux densities of Brunetti et al. (2001), and the X-ray flux densities and spectra are plotted together with the synchrotron models discussed in the text (solid line) and the predicted SSC (dot-dashed line) and CMB/IC (dotted line) emission from the hot spot at equipartition. The high-energy cutoff is set to $\gamma_{\max }=1.3 \times 10^{6}$ in this figure.

in our model. With only one high-resolution, highfrequency radio map, it is impossible to test this suggestion, but the possible offset seen between the radio and optical positions may support it.

Unlike Brunetti et al., because of the lower $R$-band optical flux density we obtain, we find $3 \mathrm{C} 351 \mathrm{~L}$ to have a relatively flat optical spectral index, $\alpha_{\mathrm{O}} \approx 0.8$ (similar to the $\mathrm{X}$-ray spectral index), and this means that a synchrotron model connecting the optical and X-ray emission cannot be ruled out in this hot spot either and is an alternative explanation for the bright $\mathrm{X}$-ray emission, although it would require a second, flat-spectrum synchrotron component to be present.

The differences we find between the equipartition/ minimum energy and SSC fields in these two hot spots are larger than the factor of $\sim 3$ inferred by Brunetti et al. (2001) because of differences in our assumptions. As noted above, we use larger angular sizes for the hot spots, and this accounts for a substantial part of the difference. Brunetti et al. used a different definition of the minimum energy, obtaining a lower field strength than our calculation would have given on the same assumptions about hot spot size. They also used a version of the more complex electron energy spectrum described by Brunetti et al. (2002a). This illustrates a general point: differences in model parameters can have an important effect on the derived magnetic field strength.

The weak south radio hot spot of $3 \mathrm{C} 351$ is not detected in the X-ray. Based on the background count rate around the hot spot region, we can set an upper limit on its X-ray flux density (Table 8). It does not lie in any of the HST fields, so no optical upper limit is available, and we do not have enough high-resolution data to comment on its radio spectrum. In the $8 \mathrm{GHz}$ maps it is resolved into two compo- nents, with flux densities of $2.2 \mathrm{mJy}$ and $1.1 \mathrm{mJy}$. Both components are extended. Using a spectral model similar to that used for hot spot $\mathrm{J}$, we find that the two components of $\mathrm{A}$ taken together would have an SSC flux density of approximately $0.2 \mathrm{pJy}$, a factor of 250 below our upper limit. Thus, even if these hot spots were brighter than their equipartition flux density by the same factor as hot spot $\mathrm{J}$, we would not have detected them in our observations. We infer the weak constraint $B \gtrsim 0.04 B_{\text {eq }}$.

\subsection{Lobes}

Both lobes of 3C 351 are detected with Chandra (Fig. 17). To extract spectra, we used two circular regions at identical distances $\left(25^{\prime \prime}\right)$ from the nucleus, centered on the lobe emission, and a matched off-source background region in a suitable position angle. There are 60 net counts in the northern lobe and 40 in the southern lobe; this is insufficient for spectral analysis of either lobe alone but allowed us to fit a model to the combined emission from both (Table 8).

Modeling the lobes as described above for 3C 263 and 3C 330 , we find a predicted $1 \mathrm{keV}$ flux density for the CMB/IC process of $0.21 \mathrm{nJy}$ for the north lobe and $0.17 \mathrm{nJy}$ for the south lobe, respectively factors of $\sim 5$ and $\sim 4$ below what is observed. If the X-ray emission from these lobes is to be produced by the $\mathrm{CMB} / \mathrm{IC}$ process, the magnetic field strength must be a factor of $2-2.5$ below equipartition. The pressures in the lobes are then $\sim 4 \times 10^{-13} \mathrm{~Pa}$.

\subsection{Extended Emission}

There is no evidence for extended, cluster-related X-ray emission in 3C 351. No additional extended model that we have fitted improves the fitting statistic significantly, and the best-fitting models contain only a few tens of counts. If we 


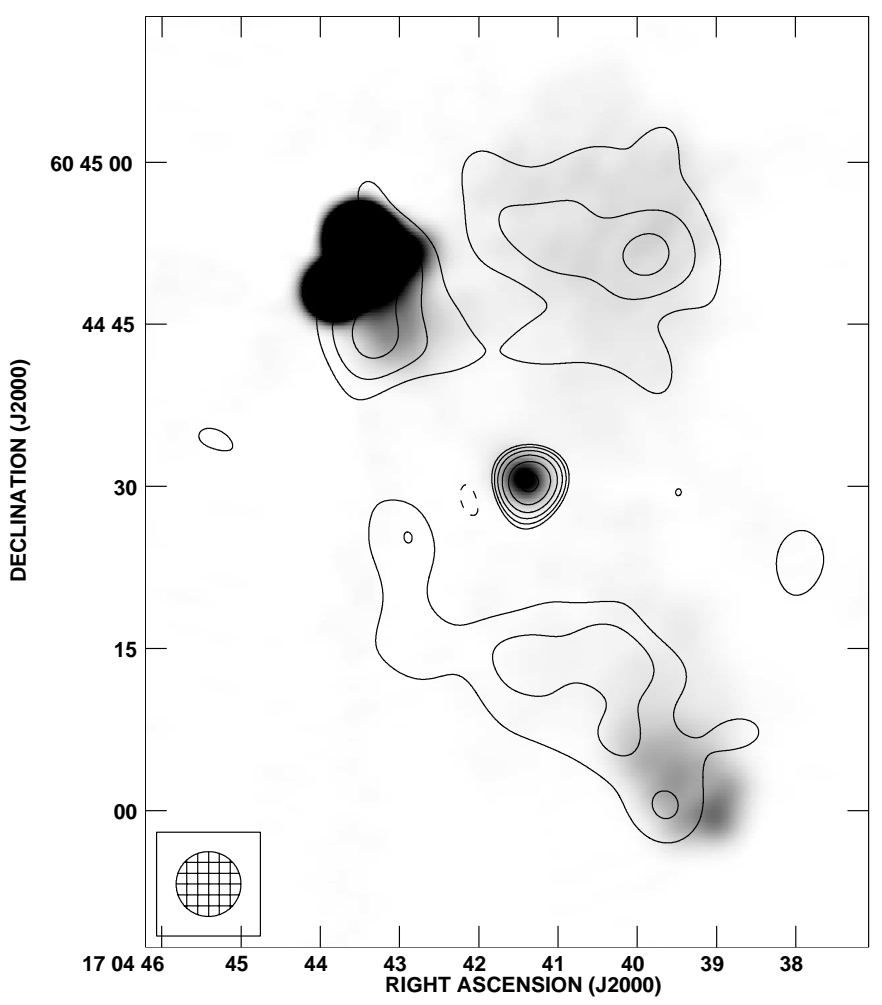

FIG. 17.- Lobes of 3C 351. The contours show the X-ray emission smoothed with a 6" (FWHM) Gaussian. The hot spot regions and background X-ray point sources were masked out before smoothing (some extended emission is present around the hot spots and gives rise to the contours near them), and a scaled, smoothed, energy-weighted PSF model was subtracted from the image after smoothing to remove the nuclear emission. A narrow peak at the core position shows that the PSF model is slightly broader than the real data. The lowest contour is the $3 \sigma$ level, defined according to the prescription of Hardcastle (2000), and the contours increase logarithmically by a factor $\sqrt{2}$. Negative contours (illustrating the residuals due to core subtraction) are dashed. The FWHM of the convolving Gaussian is shown in the bottom left of the image. The gray scale shows the $8.4 \mathrm{GHz}$ VLA image with $3^{\prime \prime}$ resolution of G02; black is $10 \mathrm{mJy}_{\text {beam }}{ }^{-1}$.

take these as very rough upper limits, the X-ray luminosity of 3C 351's environment is no more than a few times $10^{42}$ ergs $\mathrm{s}^{-1}$ (depending on choice of temperature and abundance), a group environment at best. Other evidence, such as galaxy count studies (e.g., Harvanek et al. 2001), suggests that 3C 351's environment is poor, so this is not a surprising conclusion. The gas in groups of galaxies of this sort of luminosity can have a pressure at $100 \mathrm{kpc}$ radii comparable to that estimated above for the lobes of $3 \mathrm{C} 351$, so it remains possible that 3C 351's lobes are pressure-confined by a faint $\mathrm{X}$-ray-emitting environment.

\section{DISCUSSION}

\subsection{Evidence for and against Equipartition in Hot Spots}

The inferred field strengths, relative to the equipartition values, of the hot spots of the target sources are listed in Table 10. Of the three sources, one (3C 330) has hot spots whose X-ray emission is consistent with SSC emission at equipartition, one (3C 263) has an inferred hot spot magnetic field strength a factor of 2 below the equipartition value (consistent with other sources, such as 3C 295, which show a similar deviation from equipartition), and one (3C 351) has hot spots whose X-ray emission, if it were of SSC origin, would imply a magnetic field strength an order of magnitude below the equipartition value.

What process is responsible for the anomalously bright, flat-spectrum hot spots in 3C 351? We can consider several classes of explanation. The first involves retaining the SSC process, with some modifications; the second involves invoking relativistic beaming, an idea supported by the observation of very bright, one-sided radio hot spots on the jet side of 3C 351 and by the displacement of the hot spots with respect to the radio lobe; and the third involves some emission mechanism other than the inverse Compton process. We discuss several variants of these models in turn.

1a. SSC with standard electron spectral model, $B \ll B_{\text {eq. }}$ As discussed above, this model does not explain the offsets between the radio and X-ray peaks in hot spot L.

1b. SSC with standard electron spectral model, $B \ll B_{\mathrm{eq}}$, and spatial variation of the electron population.-This model can explain the offsets between the peaks in various wavebands, at the cost of introducing new features in the electron population for which there is no independent observational evidence.

1c. SSC with second electron population, $B \sim B_{\mathrm{eq}}$.-If we introduce an additional population of low-energy electrons whose synchrotron emission is below the observed radio band, we can greatly increase the SSC flux. If we also allow this population to be offset with respect to the radioemitting population, we can explain the properties of hot spot $\mathrm{L}$. This model has the same disadvantages as model $1 \mathrm{~b}$.

1d. SSC with relativistic beaming, $B \sim B_{\mathrm{eq}}$.-Beaming would mean that our estimate of the hot spot flux density would not correspond to the source-frame value. The predicted SSC flux density and the ratio $B_{\text {eq }} / B$ depend on the Doppler factor $\mathscr{D}\left(\mathscr{D}=[\Gamma(1-\beta \cos \theta)]^{-1}\right.$ for a hot spot, where $\theta$ is the angle to the line of sight and $\Gamma=\left(1-\beta^{2}\right)^{-1 / 2}$ is the bulk Lorentz factor). Beaming causes the observed flux densities to change by a factor $\mathscr{D}^{(3+\alpha)}$. For a pure power-law spectrum with electron energy index $p$ $[\alpha=(p-1) / 2]$, neglecting aberration effects, the predicted

TABLE 10

The Ratio between Equipartition and Inferred Field Strengths in Hot Spots AND LOBES

\begin{tabular}{|c|c|c|c|c|}
\hline \multirow[b]{2}{*}{ Source } & \multicolumn{4}{|c|}{$B_{\mathrm{eq}} / B$} \\
\hline & North Hot Spot & South Hot Spot & North Lobe & South Lobe \\
\hline $3 C 263 \ldots$ & $<4$ & $1.9 \pm 0.1$ & $2.0 \pm 0.3$ & $2.0 \pm 0.4$ \\
\hline 3C $330 \ldots \ldots \ldots \ldots \ldots$ & $1.0 \pm 0.1$ & $1.5 \pm 0.4$ & $1.4 \pm 0.3$ & $1.4 \pm 0.3$ \\
\hline 3C $351 \ldots \ldots \ldots \ldots \ldots$ & $12 \pm 0.05$ & $<25$ & $2.5 \pm 0.4$ & $2.0 \pm 0.5$ \\
\hline
\end{tabular}

Note.-For 3C 351, hot spot $\mathrm{J}$ is used. Errors are based on the statistical errors on $1 \mathrm{keV}$ flux density quoted in Table 8 and do not include systematic uncertainties in the modeling. 
SSC flux can be shown to go as $\mathscr{D}^{-12 /(p+5)}$, and so, for values of $p$ in the range $2-3$, Doppler boosting, $(\mathscr{D}>1)$ reduces the predicted equipartition flux while Doppler dimming $(\mathscr{D}<1)$ increases it. To place 3C 351 at equipartition we would require $\beta \gtrsim 0.999$ ( $\Gamma \gtrsim 20$ ) with the source lying close to the plane of the sky. This is clearly not a plausible model for a quasar; in addition, the rest-frame radio luminosity of the hot spot becomes very large.

2a. Boosted $C M B, B \sim B_{\text {eq }}$.- - Relativistic fluid speeds in or close to the hot spot would give rise to an increased contribution from inverse Compton scattering of the microwave background, which is negligible in all our sources if relativistic effects are not present. The effective energy density in microwave background photons increases as $\sim \Gamma^{2}$ (e.g., Dermer \& Schlickeiser 1993), but this is partly offset by the reduction in the electron number density. There are additional corrections because of the anisotropic nature of the inverse Compton process; the net result is that a source exhibiting emission from this process must be close to the line of sight. Because of the blueshifting of the CMB photons in the source frame, the results are also strongly dependent on the presence of low-energy electrons; in order to obtain a spectrum that is not flat or inverted, we require, approximately, $2 \times 10^{-7}(1+z) \gamma_{\min }^{2} \Gamma^{2} \ll 1$. To reproduce the observed emission from 3C 351 with an equipartition $B$-field and the lowest possible bulk $\Gamma, \Gamma \sim 5$, we require $\gamma_{\min } \sim 10$ and $\theta=0^{\circ}$. Larger angles to the line of sight require larger $\Gamma$ values. This model thus requires $3 \mathrm{C} 351$ to be very close to the line of sight (for $5<\Gamma<20, \theta \lesssim 6^{\circ}$, so the linear size of the source would have to be $\gtrsim 4 \mathrm{Mpc}$, if bending is neglected). We also require highly relativistic flows to power both $\mathrm{J}$ and $\mathrm{L}$ : either the jet splits, or (more conventionally) a relativistic outflow from $\mathbf{J}$ powers $\mathrm{L}$. In the second case, we would expect $\Gamma$ to be different in the two hot spots. The angles between the line of sight and the velocity vectors of the flows powering the hot spots are likely to differ in either situation, because the hot spots and the core do not lie on a straight line: so in this model the parameters must "conspire" to ensure that the X-ray to radio ratios remain similar in the two hot spots. This model does not explain the offsets in hot spot L without some additional assumptions, such as finely tuned velocity vector variations in the hot spot.

2b. Boosted CMB, but hot spots are "jet knots," $B \sim B_{\text {eq }}$. - In this model, the objects we have described as hot spots are not terminal hot spots at all, but knots in a projected jet (which presumably terminates somewhere else (e.g., in the displaced north lobe) in a hot spot of brightness comparable to that in the south lobe. This model has the advantage that we do not (necessarily) expect jet deceleration between knots and that it allows us to retain subrelativistic speeds in the hot spots. Otherwise it has the same disadvantages as model $2 \mathrm{a}$.

2c. Boosted hot spot back-scattering, B unknown.-We cannot rule out the possibility that the X-ray emission does not originate in the hot spot (postshock) region at all. It could instead be due to scattering of hot spot photons by the incoming, presumably highly relativistic jet. This model has the advantage that it can explain the offsets observed in hot spot L. It has the disadvantage that, as no radio jets are observed entering the hot spot, we cannot use the observations to constrain magnetic fields or jet speeds. Because the IC emission from this process is even more anisotropic than for models $2 a$ and $2 b$, the problem of the angle made by the jet to the line of sight becomes greater in this model.

3a. Synchrotron from second electron population, $B$ unknown.-A second, flat-spectrum synchrotron component in the X-ray (and possibly also the optical) can explain many of the observations of 3C 351's hot spots, including the offsets seen in hot spot L if the second electron population is spatially offset from the low-energy electrons responsible for the radio emission. Given the very different loss timescales in the radio and X-ray, and the rapidly changing nature of hot spots (and the bulk flows that feed them) that numerical simulations suggest, there is no very strong reason to believe that a single electron energy power law should describe observations made at a given moment, so a picture of this kind is not inconsistent with standard models of radio sources. The $\mathrm{X}$-ray emission in the jets of certain sources, such as 3C 120 (Harris et al. 1999) and 3C 273 (Jester et al. 2002), has been explained in terms of a model of this kind.

3b. Synchrotron from second electron population and SSC, $B$ unknown but $B \sim B_{\text {eq }}$ permitted.-This is a trivial variation on model $3 \mathrm{a}$ in which some of the $\mathrm{X}$-ray emission is due to SSC rather than synchrotron.

3c. Proton-induced cascade or other exotica, $B$ unknown.-As usual, we cannot rule out the possibility that some less familiar emission process makes a contribution to the X-ray emission.

All these models are ad hoc in one way or another. The beaming models do help to explain the large-scale asymmetry of the radio source as well as the X-ray emission, but they all require $3 \mathrm{C} 351$ to be very close to the line of sight and extremely large in unprojected linear size, as well as invoking speeds in the hot spot regions that are very much higher than have been inferred from other work. The other models require less extreme parameters for the radio source but do not answer the question of why 3C 351 is unlike the other sources in the sample, which show no evidence for strong departures from equipartition, extra electron populations, or new emission mechanisms.

It is interesting to compare the results on 3C 351 with those on other FR II sources with "bright" X-ray hot spots, such as Pictor A and 3C 390.3. In the case of Pictor A, Wilson, Young, \& Shopbell (2001) found that a magnetic field a factor of 14 below the equipartition value would reproduce the flux density of the X-ray hot spot, but they reject a simple SSC model of the type we have used in this paper because of the well-constrained steep spectrum of the hot spot $\left(\alpha_{X}=1.07 \pm 0.11\right)$. A simple synchrotron model is also rejected because the optical data require a cutoff in the optical (Meisenheimer, Yates, \& Röser 1997). However, Wilson et al. do not rule out a model in which there are contributions from both X-ray synchrotron and SSC emission, as in model $3 \mathrm{~b}$ above, which requires $B_{\mathrm{eq}} / B \approx 9$. This factor is similar to those that we would require in $3 \mathrm{C} 351$ on the pure SSC model (1a). They also consider the possibility of a second electron population (models $1 \mathrm{c}$ and $3 \mathrm{a}$ above).

In the case of 3C 390.3, Harris et al. (1998) rejected a SSC model for the ROSAT-detected X-ray hot spot, hot spot B, because the X-ray emission was much brighter than their SSC prediction. The Chandra observation of this source (S. Wagner et al., in preparation) detects all three compact hot spots in 3C 390.3 and allows a more precise determination of some of the source parameters. Examining the 
archive data, we find that the brightest hot spot, hot spot B, has a $1 \mathrm{keV}$ flux density of $4.5 \pm 0.3 \mathrm{nJy}$, in good agreement with the result of Harris et al., and $\alpha_{\mathrm{X}}=0.88 \pm 0.17$. The radio, optical, and $\mathrm{X}$-ray data in hot spot $\mathrm{B}$ can be connected with a simple synchrotron model. An SSC model requires a large departure from equipartition; with our cosmology and standard spectral assumptions we find $B_{\text {eq }} / B \approx 90$, much higher than in any other source we have examined. Moreover, the predicted SSC spectral index is $\sim 0.5$, while the spectral index in the synchrotron model is $\sim 1.0$, so that the Chandra spectrum favors the synchrotron model. We see no reason to believe that there is a significant contribution from SSC emission in hot spot B of 3C 390.3. It seems likely that the differences between the radio to X-ray spectra of the three hot spots, remarked on by Harris et al., can be explained in terms of similar electron spectra with different high-energy cutoffs. Given the large number of jets in low-power radio galaxies now thought to be due to synchrotron radiation $(\S 1)$, the existence of the required high-energy electrons in hot spots is no longer as remarkable as it once seemed. This observational evidence for synchrotron emission in hot spots is indirect support for one of the synchrotron models ( $3 a$ and $3 b$ above) to explain the emission from 3C 351.

The Chandra data on hot spots in general are therefore reinforcing the conclusion, already suggested by ROSAT observations, that more than one emission mechanism is operating in the X-ray in FR II hot spots. Some hot spots, of which 3C 390.3 is to date the most convincing example, require an X-ray synchrotron model. Some, such as Cygnus A, 3C 123, 3C 263, and 3C 330, are likely to be pure SSC, with no significant contribution to the $\mathrm{X}$-rays from synchrotron emission and a hot spot magnetic field close to the equipartition value. And some, such as Pictor A and perhaps $3 \mathrm{C}$ 351 (model 3b above), may have synchrotron and SSC emission at similar levels, or may be different from the other sources in some more interesting way. In order to distinguish between these cases, good radio and optical information and, ideally, an X-ray spectrum of the hot spot are required: further high-resolution observations in the radio, optical and UV may be required to disentangle the emission mechanisms in Pictor A and 3C 351.

In this picture, the questions remaining to be answered are the following:

1. What is special about the hot spots that exhibit X-ray synchrotron emission? It is particularly striking that all three compact hot spots of 3C 390.3 are X-ray synchrotron emitters, despite being quite different in their local environments and radio structures (see Leahy \& Perley 1995). There is no obvious way in which these hot spots, in quite different parts of the source, can "know" that they should accelerate electrons to high energies - unless some intrinsic property of the beam is involved.

2. What is special about the hot spots, like 3C 351 and possibly Pictor A, that may have $B_{\text {eq }} / B \gg 1$ ?

Brunetti et al. (2001) suggested a partial answer to the second of these questions. They pointed out that, in standard shock-acceleration models, the high-energy cutoff of the synchrotron electron population is higher for lower hot spot magnetic fields, because it is determined by the balance between acceleration and synchrotron loss processes. In other words, we might expect to see an enhanced contribution from SSC emission in radio sources that we know by other means to have a synchrotron spectrum extending to high energies. This might apply to 3C 351 and Pictor A, which are known to have optical synchrotron hot spots, while sources like Cygnus A and 3C 123, whose electron spectra are known to cut off at lower energies, have fields closer to equipartition. However, one source in the current sample, 3C 263, breaks the trend by having an optical synchrotron hot spot without being particularly X-ray-bright. And this picture does not explain why it is that some sources have particularly low hot spot B fields, or why, in the case of 3C 351, both hot spots appear to do so.

Numerical simulations (e.g., Tregillis, Jones, \& Ryu 2001) lead us to expect that hot spots will be transient structures and may well have different magnetic field and electron acceleration behavior at different times. All hot spots may pass through phases of inverse Compton domination and synchrotron domination of their X-ray output. If this is the case, neglecting the probably important question of observational bias, then the statistics of detected sources are telling us that sources spend approximately equal times in the two regimes. However, this does not explain (and is even to some extent incompatible with) the observation of correlated properties in spatially separated hot spots, as observed in Cygnus A, 3C 351 and 3C 390.3.

\subsection{Inverse Compton Emission from Lobes? Constraints on Lobe Physics}

Both lobes of all three sources are detected in the $\mathrm{X}$-ray. In all three cases, the measured X-ray emission is close to the predicted value for inverse Compton scattering of $\mathrm{CMB}$ photons, requiring field strengths that are factors between 1.4 and 2.5 below equipartition values on simple models that treat the lobes as uniform (Table 10). We have not taken into account any possible illumination of the lobes by the quasar nuclei (nuclear inverse Compton, or NIC), which would give an additional photon contribution and reduce the discrepancy between the inferred and equipartition magnetic field strengths. We do not believe that nuclear illumination is likely to dominate the CMB photon field in our sources, since in $3 \mathrm{C}$ 263 and 3C 351 there is no evidence for a higher ratio of $\mathrm{X}$-ray to radio flux in the lobes on the counterjet side, as would be expected, due to the anisotropy of the inverse Compton process, in a NIC model (Brunetti, Setti, \& Comastri 1997).

Is the lobe emission inverse Compton in origin at all? The similarity of the observed fluxes to the predicted values, and the tight relationship between the emitting regions in radio and X-ray, suggest this, but cannot confirm it. The X-ray spectra are not good enough in any of our sources to allow us to rule out a thermal origin for the lobe emission, although the existence of some hard counts (e.g., in 3C 263's north lobe and 3C 351) would imply quite high temperatures, $k T \sim 5 \mathrm{keV}$ ). If the $\mathrm{X}$-rays were thermal, their spatial similarity to the radio emission means that they would have to originate either inside the lobes, or in a boundary layer around the lobes with a thickness considerably less than the lobe dimensions (i.e., no more than a few kiloparsecs, given the spatial resolution of Chandra). Polarization observations are conventionally taken to rule out the presence of large amounts of thermal material inside radio lobes, although, as pointed out by Laing (1984), large amounts of thermal material can be hidden by suitable magnetic field 
configurations. Unfortunately, estimates of the depolarization measure DP are not available to us for either of our quasars. For 3C 330, Fernini (2001) quotes values of DP (the ratio of polarization at two frequencies, in this case 1.4 and $5 \mathrm{GHz}$ ) of 0.84 and 0.55 for the two lobes. (A lower DP means more depolarization.) We can convert the observed Chandra count rate for the lobes of 3C 330 into a thermal proton density using XSPEC and assuming that the thermal plasma uniformly fills the radio lobes; we use a mekal model $^{5}$ with $k T=5 \mathrm{keV}, 0.3$ solar abundance and Galactic absorption. We find that a thermal proton density of $\sim 10^{4}$ $\mathrm{m}^{-3}$ would be required. Using equipartition fields, this would give rise to a large Faraday depth, and, using the results of Cioffi \& Jones (1980), we would expect $\mathrm{DP} \sim 0.08$, which is much less than the observed value; we would also expect a very low degree of polarization at low frequencies, whereas we know that the source remains polarized at $1.4 \mathrm{GHz}$. We cannot reduce the Faraday depth by significantly decreasing the assumed magnetic field strength, as the X-ray observations set a lower limit on the field strength, $B>B_{\text {eq }} / 1.5$. So, for simple field geometries, the idea that thermal protons are inside the lobes is not consistent with the radio data.

If the thermal protons are instead in a boundary layer of shocked gas around the source, their density can be estimated in the same way, and is greater than the value calculated above by the square root of the ratio of the boundary layer to source volumes: for example, if the boundary layer were $0.5(3.5 \mathrm{kpc})$ thick in $3 \mathrm{C} 330$, the density would be higher by a factor of $\sim 1.5$. Here Faraday-rotation constraints are less helpful, because we do not know the value of the external magnetic field strength. However, if the external thermal material is assumed to be shocked ambient gas, well-known results for the density contrast in a shock imply that the ambient gas density must be no more than a factor of 4 less dense than the inferred external density, or at least a few times $10^{3} \mathrm{~m}^{-3}$. This is comparable to, although on average somewhat larger than, the density of the external material inferred from the observations described in $\S 5.5$ at the distance of the lobes of $3 \mathrm{C} 330$, so this model remains possible from that perspective. However, it seems likely that this external gas is too hot (particularly in 3C 263) to give rise to the observed X-ray emission when shocked, unless the shocks are only weak (in which case the stand-off distance needs to be large). There is also no obvious gradient of X-ray emissivity along the sources, particularly 3C 330, although the $\beta$-model fits of $\S 5.5$ imply that the external density drops by an order of magnitude along the length of the lobes in that source. And there is no evidence in the $\mathrm{X}$-ray data of the limb-brightening expected in such a model, although the small number of counts from the lobes makes detailed analysis of the lobe structure difficult. We conclude that, although models in which the lobe-related $\mathrm{X}$-ray emission are due to an external boundary layer are not ruled out, they are more contrived than the alternative of an inverse Compton origin with a magnetic field somewhat lower than the equipartition value.

\footnotetext{
${ }^{5}$ This model includes both thermal bremsstrahlung and line emission from a hot optically thin plasma and is generally found to be a good fit to high signal-to-noise observations of clusters.
}

Whether or not the emission is inverse Compton in origin, its detection places some interesting constraints on the filling factors in the lobes, by giving an upper limit on lobe X-ray emissivity. As pointed out by Hardcastle \& Worrall (2000), the dependence of inverse Compton emissivity on filling factor is complicated, depending on the nature of the "fluid" that fills the gaps between radio-emitting regions. However, if the fluid is relativistic electrons, i.e., the lobes consist of a more or less uniform distribution of electrons with a very wide range in magnetic field strengths, then the inverse Compton emissivity increases as the filling factor $\phi$ decreases. In the simple case in which the magnetic field is either on or off, and where on is in energy equipartition with the synchrotronemitting electrons, the IC emissivity over the entire lobe goes up approximately as $\phi^{-4 / 7}$. This sort of model is constrained by the observations to have $\phi \gtrsim 0.1$. The constraint is difficult to evade if we require that there are no strong pressure differences in the lobes. Filling factors lower than this are only possible if the nonradiating "fluid" does not consist of electrons-for example, if the magnetic field dominates the overall energy of the lobes. We note that less extreme models with inhomogenous magnetic field distributions are not ruled out by the data. For example, the models used by Tribble (1991) give only a modest increase $(\sim 20 \%)$ in the expected inverse Compton emissivity if the mean field energy is in equipartition with the electrons.

A CMB inverse Compton model and the inferred relatively small but nonzero departures from equipartition are consistent with early observations of less powerful sources with ROSAT and ASCA (e.g., Feigelson et al. 1995; Tsakiris et al. 1996; Tashiro et al. 1998), as well as with new results now emerging from Chandra observations of sources more similar to our targets (Brunetti et al. 2002a, 2002b; Fabian, Celotti, \& Johnstone 2002).

\subsection{Thermal Emission and Pressure Balance}

The extended emission around 3C 263 and 3C 330 seems very likely to be thermal emission from a cluster-scale atmosphere: we have thus confirmed earlier predictions (Hall et al. 1995) that 3C 263 should lie in an X-ray-emitting cluster. If the lobe X-rays are indeed inverse Compton emission, the model we favor of those discussed in $\S 7.2$ above, then the required departures from equipartition produce lobe pressures which are consistent, within the uncertainties on the various calculations, with the inferred external pressures. Small departures from equipartition may therefore solve the problem, discussed by Hardcastle \& Worrall (2000), of the apparent underpressuring of the radio lobes with respect to the external medium; this is particularly true if some of the X-ray emission detected by Hardcastle \& Worrall with ROSAT was in fact inverse Compton emission from the lobes of their target sources. More observations are required to test this hypothesis. We note, however, that our observations provide little or no support for the idea that lobes are strongly overpressured with respect to the external medium. For this to be the case, there would need to be another contribution to the internal lobe pressure, for example from relativistic protons, which cannot be detected with the present observations. 


\section{CONCLUSIONS}

We have detected X-ray emission from the hot spots and lobes of three powerful FR II radio sources, and low-level extended emission from the hot-gas environments of two of them.

The hot spots in two of the objects we have studied, 3C 330 and 3C 263, are consistent with being SSC emission with a hot spot magnetic field within a factor of 2 below the value for energy equipartition with the synchrotron-emitting electrons. We argue therefore that magnetic field strengths close to equipartition are common in hot spots.

The double hot spots in the third source, 3C 351, are difficult to accommodate within an SSC model; they require magnetic field strengths much further from equipartition and, at least in one hot spot, there are offsets between the radio and X-ray centroids that a simple SSC model does not explain. The flat X-ray spectral indices rule out the possibility that the X-rays can be synchrotron emission from the electron population responsible for the radio emission. We are left with several alternative models, none of them particularly attractive: the most plausible contenders are an SSC model with large departures from equipartition, together with unmodeled spatial structure in the electron population in the secondary hot spot, a synchrotron model with a second, highenergy electron population, or strong beaming effects in the hot spot regions. In any case, it is striking that the two hot spots behave in such a similar way, in spite of their large spatial separation (and the expectation that primary and secondary hot spots should have different physics). As we have pointed out, other X-ray hot spot sources, like Cygnus A and 3C 390.3, seem to share this tendency for the hot spots to appear to know about each other.
The lobe emission we have detected seems most likely to be due to inverse Compton scattering of microwave background photons by the synchrotron-emitting electron population. All three sources can be explained by such a model, in which the magnetic field strengths in the lobes are at most a factor of 2 below equipartition (on a simple model with uniform field and electron distributions in the lobes). If this model is correct, the pressures in lobes are a factor of a few above the canonical minimum pressures. This factor may be enough to account for the discrepancy between the minimum pressure and the pressure in the external hot gas environment, discussed by Hardcastle \& Worrall (2000), and indeed we suggest that the two sources with detected extended X-ray emission, 3C 263 and 3C 330, are in approximate pressure balance with the hot gas in their cluster/ group environments; such a model is also possible for 3C 351 .

We are grateful to Gianfranco Brunetti for helpful discussions of inverse Compton scattering and to an anonymous referee for comments that improved the presentation of the paper. The work at SAO was partially supported by NASA grants and contracts GO1-2109X and NAS 8-39073. The National Radio Astronomy Observatory is a facility of the National Science Foundation operated under cooperative agreement by Associated Universities, Inc. We are grateful to the VLA schedulers for allowing us to make an observation of 3C 263 at short notice and to the data analysts for supporting our archive requests. We thank George Gilbert, Robert Laing, Laura Mullin, and Julia Riley for providing us with radio maps and $u v$ data at various stages in this project, and Hugh Aller, Wil van Breugel, Robert Laing, and Bev Wills for allowing us to use their VLA archival observations.
Arshakian, T. G., \& Longair, M. S. 2000, MNRAS, 311, 846

Best, P. N., Bailer, D. M., Longair, M. S., \& Riley, J. M. 1995, MNRAS, 275,1171

Birkinshaw, M., \& Worrall, D. M. 1993, ApJ, 412, 568

Bridle, A. H., Hough, D. H., Lonsdale, C. J., Burns, J. O., \& Laing, R. A. 1994, AJ, 108, 766

Brunetti, G. 2000, Astropart. Phys., 13, 107

Brunetti, G., Bondi, M., Comastri, A., Pedani, M., Varano, S., Setti, G., \& Hardcastle, M. J. 2001, ApJ, 561, L157

Brunetti, G., Bondi, M., Comastri, A., \& Setti, G. 2002a, A\&A, 381, 795

Brunetti, G. Comastri, A. Dallacasa, D., Bondi, M. Pedani, M., \& Setti, G. 2002b, in ESA Conf. Ser., New Visions of the X-Ray Universe in the $X M M$-Newton and Chandra Era, ed. F. Jansen et al., in press (astro-ph/ 0202373)

Brunetti, G., Setti, G. \& Comastri, A. 1997, A\&A, 325, 898

Burbidge, G. 1956, ApJ, 124, 416

Carilli, C. L., Perley, R. A., Dreher, J. W., \& Leahy, J. P. 1991, ApJ, 383, 554

Cavaliere, A., \& Fusco-Femiano, R. 1978, A\&A, 70, 677

Cioffi, D. F., \& Jones, T. W. 1980, AJ, 85, 368

Davis, J. E. 2001, ApJ, 562, 575

Dennett-Thorpe, J., Bridle, A. H., Laing, R. A., Scheuer, P. A. G., \& Leahy, J. P. 1997, MNRAS, 289, 753

Dermer, C. D., \& Schlickeiser, R. 1993, ApJ, 416, 458

Fabian, A. C., Celotti, A., \& Johnstone, R. M. 2002, MNRAS, submitted (astro-ph/020488)

Feigelson, E. D., Laurent-Muehleisen, S. A., Kollgaard, R. I., \& Fomalont, E. 1995, ApJ, 449, L149

Fernini, I. 2001, AJ, 122,83

Fernini, I., Burns, J. O., \& Perley, R. A. 1997, AJ, 114, 2292

Fiore, F., Elvis, M., Mathur, S., Wilkes, B., \& McDowell, J. C. 1993, ApJ, 415,129

Gilbert, G., Riley, J. M., Pooley, G. G., Alexander, P., \& Hardcastle, M. J. 2002, MNRAS, submitted

Hall, P. B., Ellingson, E., Green, R. F., \& Yee, H. K. C. 1995, AJ, 110, 513

Hardcastle, M. J. 2000, A\&A, 357, 884

2001, A\&A, 373, 881

\section{EFERENCES}

Hardcastle, M. J., Birkinshaw, M., \& Worrall, D. M. 1998, MNRAS, 294, 615

\section{1a, MNRAS, 323, L17}

2001b, MNRAS, 326, 1499

Hardcastle, M. J., \& Worrall, D. M. 1999, MNRAS, 309, 969 2000, MNRAS, 319, 562

Harris, D. E., Carilli, C. L., \& Perley, R. A. 1994, Nature, 367, 713

Harris, D. E., Hjorth, J., Sadun, A. C., Silverman, J. D., \& Vestergaard, M. 1999, ApJ, 518, 213

Harris, D. E., \& Krawczynski, H. 2002, ApJ, 565, 244

Harris, D. E., Leighly, K. M., \& Leahy, J. P. 1998, ApJ, 499, L149

Harris, D. E., et al. 2000, ApJ, 530, L81

Harvanek, M., Ellingson, E., Stocke, J. T., \& Rhee, G. 2001, AJ, 122, 2874

Heavens, A. F., \& Meisenheimer, K. 1987, MNRAS, 225, 335

Hill, G. J., \& Lilly, S. J. 1991, ApJ, 367, 1

Jester, S., Röser, H.-J., Meisenheimer, K., \& Perley, R. 2002, A\&A, 385, L27

Lähteenmäki, A., \& Valtaoja, E. 1999, AJ, 117, 1168

Laing, R. A. 1984, in Physics of Energy Transport in Radio Galaxies, ed. A. H. Bridle \& J. A. Eilek (Green Bank: NRAO), 90

Laing, R. A., Riley, J. M., \& Longair, M. S. 1983, MNRAS, 204, 151

Leahy, J. P., Muxlow, T. W. B., \& Stephens, P. W. 1989, MNRAS, 239, 401

Leahy, J. P., \& Perley, R. A. 1991, AJ, 102, 537 1995, MNRAS, 277, 1097

Lockman, F. J., \& Savage, B. D. 1995, ApJS, 97, 1

Looney, L. W., \& Hardcastle, M. J. 2000, ApJ, 534, 172

Malaguti, G., Bassani, L. \& Caroli, E. 1994, ApJS, 94, 517

Mathur, S., Wilkes, B., Elvis, M., \& Fiore, F. 1994, ApJ, 434, 493

Meisenheimer, K., Röser, H.-J., Hiltner, P. R., Yates, M. G., Longair, M. S., Chini, R., \& Perley, R. A. 1989, A\&A, 219, 63

Meisenheimer, K., Yates, M. G., \& Röser, H.-J. 1997, A\&A, 325, 57

Nicastro, F., Fiore, F., Perola, G. C., \& Elvis, M. 1999, ApJ, 512, 136

Prieto, M. A. 1997, MNRAS, 284, 627

Röser, H.-J. 1989, in Hot Spots in Extragalactic Radio Sources, ed. K. Meisenheimer \& H.-J. Röser (Heidelberg: Springer), 91

Röser, H.-J., \& Meisenheimer, K. 1987, ApJ, 314, 70

Scheuer, P. A. G. 1995, MNRAS, 277, 331 
Schwartz, D. A., et al. 2000, ApJ, 540, L69

Stark, A. A., Gammie, C. F., Wilson, R. W., Bally, J., Linke, R. A., Heiles, C., \& Hurwitz, M. 1992, ApJS, 79, 77

Tashiro, M., et al. 1998, ApJ, 499, 713

Tavecchio, F., Maraschi, L., Sambruna, R. M., \& Urry, C. M. 2000, ApJ, 544, L23

Tregillis, I. L., Jones, T. W., \& Ryu, D. 2001, ApJ, 557, 475

Tribble, P. C. 1991, MNRAS, 253, 147

Tsakiris, D., Leahy, J. P., Strom, R. G., \& Barber, C. R. 1996, in IAU Symp. 175, Extragalactic Radio Sources, ed. R. D. Ekers, C. Fanti, \& L. Padrielli (Dordrecht: Kluwer), 256
Ueno, S., Koyama, K., Nishida, M., Yamauchi, S., \& Ward, M. J. 1994, ApJ, 431, L1

Welch, W. J., et al. 1996, PASP, 108, 93

Wilson, A. S., Young, A. J., \& Shopbell, P. L. 2000, ApJ, 544, L27 2001, ApJ, 547, 740

Worrall, D. M., Birkinshaw, M., \& Hardcastle, M. J. 2001a, MNRAS, 326, L7

Worrall, D. M., Birkinshaw, M., Hardcastle, M. J., \& Lawrence, C. R. 2001b, MNRAS, 326, 1127

Wu, X.-P., Xue, Y.-P., \& Fang, L.-Z. 1999, ApJ, 524, 22 